\title{
Quantum corrections to the stress-energy tensor in thermodynamic equilibrium with acceleration
}

\author{
F. Becattini ${ }^{1}$ and E. Grossi ${ }^{1}$ \\ ${ }^{1}$ Università di Firenze and INFN Sezione di Firenze, Florence, Italy
}

\begin{abstract}
We show that the stress-energy tensor has additional terms with respect to the ideal form in states of global thermodynamic equilibrium in flat spacetime with non-vanishing acceleration and vorticity. These corrections are of quantum origin and their leading terms are second order in the gradients of the thermodynamic fields. Their relevant coefficients can be expressed in terms of correlators of the stress-energy tensor operator and the generators of the Lorentz group. With respect to previous assessments, we find that there are more second order coefficients and that all thermodynamic functions including energy density receive acceleration and vorticity dependent corrections. Notably, also the relation between $\rho$ and $p$, that is the equation of state, is affected by acceleration and vorticity. We have calculated the corrections for a free real scalar field - both massive and massless - and we have found that they increase, particularly for a massive field, at very high acceleration and vorticity and very low temperature. Finally, these non-ideal terms depend on the explicit form of the stress-energy operator, implying that different stress-energy tensor of the scalar field - canonical or improved - are thermodynamically inequivalent.
\end{abstract}

\section{INTRODUCTION}

It is common wisdom that the form of the relativistic stress-energy tensor in a thermodynamic equilibrium state has the ideal form:

$$
T^{\mu \nu}=(\rho+p) u^{\mu} u^{\nu}-p g^{\mu \nu}
$$

where $\rho$ and $p$ are the energy density and pressure, thermodynamic functions of temperature $T$ and chemical potential $\mu$, and $u$ a constant four-velocity. In quantum statistical mechanics, the above expression corresponds to the renormalized mean value 1 of the quantum stress-energy tensor operator, built from local quantum fields, with the density operator:

$$
\widehat{\rho}=(1 / Z) \exp [-\beta \cdot \widehat{P}+\zeta \widehat{Q}]
$$

where $\beta=(1 / T) u$ is a constant inverse temperature four-vector (or, simply, four-temperature), with $T=1 / \sqrt{\beta^{2}}$ being the proper (or comoving) temperature and $u$ the constant four-velocity, $\zeta=\mu / T$ is the ratio between proper chemical potential and proper temperature, $\widehat{P}$ the four-momentum operator and $\widehat{Q}$ an internal conserved charge:

$$
T^{\mu \nu}(x)=\operatorname{tr}\left(\widehat{\rho} \widehat{T}^{\mu \nu}(x)\right)_{\mathrm{ren}}=\frac{1}{Z} \operatorname{tr}\left(\widehat{T}^{\mu \nu}(x) \exp [-\beta \cdot \widehat{P}+\zeta \widehat{Q}]\right)_{\mathrm{ren}}=(\rho+p) u^{\mu} u^{\nu}-p g^{\mu \nu}
$$

The form (2) is dictated by the symmetries of the density operator (1) which is traslationally invariant and isotropic in the rest frame where $\beta=(1 / T)(1, \mathbf{0})$.

However, the density operator (1), is not the only form of global thermodynamic equilibrium, which is, in general, a state where the entropy $S=-\operatorname{tr}(\widehat{\rho} \log \widehat{\rho})$ is constant. For instance, it is well known [1, 2] that in non-relativistic quantum mechanics the operator:

$$
\widehat{\rho}=(1 / Z) \exp \left[-\widehat{H} / T_{0}+\omega \widehat{J}_{z} / T_{0}\right]
$$

where $T_{0}$ is a constant global temperature ${ }^{2}, \widehat{H}$ the hamiltonian and $\widehat{J}_{z}$ the angular momentum operator along some axis $z$, represents a globally equilibrated spinning fluid with angular velocity $\omega$. Similarly (see sect. III) the operator:

$$
\widehat{\rho}=(1 / Z) \exp \left[-\widehat{H} / T_{0}+a \widehat{K}_{z} / T_{0}\right]
$$

$\widehat{K}_{z}$ being the generator of a Lorentz boost along the $z$ axis, represents a relativistic fluid with constant comoving acceleration along the $z$ direction and it is still an equilibrium distribution. These two cases belong to a more general

\footnotetext{
${ }^{1}$ For free quantum fields, by renormalization we mean the use of normal ordering in the stress-energey tensor operator

2 The global temperature $T_{0}$ is a temperature measured by a thermometer at rest with the external observer. In general, it differs from the proper temperature $T$ measured by a comoving thermometer
} 
class of thermodynamic equilibria which, in special relativity, are characterized by a four-temperature $\beta(x)$ field fulfilling the equation

$$
\partial_{\mu} \beta_{\nu}+\partial_{\nu} \beta_{\mu}=0
$$

which means that the four-temperature is a Killing vector field. We will show how for such thermodynamic equilibrium states, with the appropriate treatment in quantum relativistic statistical mechanics, the ideal form of the stress-energy tensor gets quantum corrections - vanishing in the $\hbar \rightarrow 0$ limit - whose leading terms are proportional to the squared gradients of $\beta$, which in turn can be expressed in terms of the acceleration $a^{\mu}$ and the vorticity $\omega^{\mu}$ fields (see sect. IV] for definitions):

$$
T^{\mu \nu}=(\rho+p) u^{\mu} u^{\nu}-p g^{\mu \nu}+\hbar^{2}\left(\mathcal{O}\left(a^{2}\right)+\mathcal{O}\left(\omega^{2}\right)+\mathcal{O}(a \omega)\right)
$$

As we will see, these corrections are normally tiny but they can become relevant under specific circumstances and, moreover, they are not microscopic in the sense of being relevant only at very small scales.

The appearance of these terms is somehow in contrast to the widespread belief that deviations from the ideal form (2) can only arise in presence of dissipative processes. In fact, the existence of such terms has been pointed out by a classification of second order gradient corrections of the stress-energy tensor in conformal hydrodynamics [3, 4] also by means of kinetic theory [5] and some coefficients, denoted as thermodynamic in view of their survival at equilibrium, have been calculated in ref. [6] for conformal field theories.

In this paper, we show that the occurrence of non-dissipative corrections to the ideal form of the stress-energy tensor is a general fact which is related to the very notion of equilibrium in quantum relativistic statistical mechanics. Moreover, these corrections result from the expansion of the density operator and their form is not assumed a priori like in the Landau-frame based gradient expansion. At equilibrium, they have simple and suggestive expressions as correlators of the stress-energy tensor with the generators of the Lorentz group. The proper energy density expression is also modified, as well as the relation between energy density and pressure, that is the equation of state. It is an almost straigthforward consequence that these corrections will extend to a curved spacetime.

The paper is organized as follows: in section [I] we obtain the form of the density operator of general thermodynamic equilibrium in quantum statistical mechanics in flat spacetime. In section III we discuss the relation between local observables and the local value of the four-temperature field. In section IV we derive the form of the corrections to the ideal form of the stress-energy tensor as a perturbative expansion. In section $\nabla$ we calculate those quantum corrections in free scalar field theory. Finally, in sections VI and VII we discuss the most important physical consequences and draw the conclusions.

\section{Notation}

In this paper we use the natural units, with $\hbar=c=K=1$.

The Minkowskian metric tensor is $\operatorname{diag}(1,-1,-1,-1)$; for the Levi-Civita symbol we use the convention $\epsilon^{0123}=1$. We will use the relativistic notation with repeated indices assumed to be summed over, however contractions of indices will be sometimes denoted with dots, e.g. $u \cdot T \cdot u \equiv u_{\mu} T^{\mu \nu} u_{\nu}$. Operators in Hilbert space will be denoted by a large

upper hat, e.g. $\widehat{T}$ while unit vectors with a small upper hat, e.g. $\hat{v}$. The stress-energy tensor is assumed to be symmetric with an associated vanishing spin tensor.

\section{EQUILIBRIUM IN RELATIVISTIC QUANTUM STATISTICAL MECHANICS}

A general covariant form of the density operator in relativistic quantum statistical mechanics extending the eq. (1) was first proposed, to our knowledge, in refs. [7, 8]:

$$
\widehat{\rho}=\frac{1}{Z} \exp \left[-\int_{\Sigma} \mathrm{d} \Sigma_{\mu}\left(\widehat{T}^{\mu \nu} \beta_{\nu}-\zeta \widehat{j}^{\mu}\right)\right]
$$

where $\Sigma$ is a spacelike 3D hypersurface. This form can be obtained maximizing the total entropy with the constraints of given energy-momentum and charge densities at some specific "time" of the hypersurface $\Sigma$, see the detailed discussions in ref. [8] and more recently in refs. [9, 10. The density operator (6) is therefore especially suitable to describe local thermodynamic equilibrium - that is a situation where the thermodynamic parameters temperature, velocity field and chemical potential are a function of space and time - in a quantum relativistic framework. The operator (6) will not maintain its form under the unitary time evolution and cannot thus represent the actual quantum state 
in the Heisenberg representation. However, it is time independent or, equivalently, independent of the integration hypersurface $\Sigma$ if the divergence of the integrand vanishes and in this case the (6) is the density operator of a thermodynamic equilibrium state. For conserved stress-energy tensor and current this condition leads to the request [1] that $\zeta$ is a constant and $\beta$ a Killing vector field fulfilling eq. (5) (with partial derivatives replaced by covariant derivatives if necessary).

The density operator (6) is also well suited to describe thermodynamic equilibrium in a general curved spacetime possessing a timelike Killing vector field. In Minkowski spacetime, which we will be dealing with in this work, the general solution of the eq. (5) is:

$$
\beta^{\nu}=b^{\nu}+\varpi^{\nu \mu} x_{\mu}
$$

where $b$ is a constant four-vector and $\varpi$ a constant antisymmetric tensor, which, because of (7) can be written as an exterior derivative of the $\beta$ field:

$$
\varpi_{\nu \mu}=-\frac{1}{2}\left(\partial_{\nu} \beta_{\mu}-\partial_{\mu} \beta_{\nu}\right)
$$

Hence, the general equilibrium form in flat spacetime of the density operator 6 reads:

$$
\widehat{\rho}=\frac{1}{Z} \exp \left[-b_{\mu} \widehat{P}^{\mu}+\frac{1}{2} \varpi_{\mu \nu} \widehat{J}^{\mu \nu}+\zeta \widehat{Q}\right]
$$

where the $\widehat{J}$ 's are the generators of the Lorentz transformations:

$$
\widehat{J}^{\mu \nu}=\int_{\Sigma} \mathrm{d} \Sigma_{\lambda}\left(x^{\mu} \widehat{T}^{\lambda \nu}-x^{\nu} \widehat{T}^{\lambda \mu}\right)
$$

Therefore, besides the chemical potentials, the most general equilibrium density operator in Minkowski spacetime can be written as a linear combinations of the 10 generators of the Poincaré group with 10 constant coefficients. The most widely known case is the one with $\beta=b$ and $\varpi=0$, that is eq. (1), what we define as homogeneous thermodynamic equilibrium. The rotating global equilibrium in eq. (3) can be obtained as a special case of eq. (9) setting:

$$
b_{\mu}=\left(1 / T_{0}, 0,0,0\right) \quad \varpi_{\mu \nu}=\left(\omega / T_{0}\right)\left(g_{1 \mu} g_{2 \nu}-g_{1 \nu} g_{2 \mu}\right)
$$

where $\omega$ has the meaning of a costant angular velocity [1]. Similarly, the form 4 can be obtained by setting:

$$
b_{\mu}=\left(1 / T_{0}, 0,0,0\right) \quad \varpi_{\mu \nu}=\left(a / T_{0}\right)\left(g_{0 \mu} g_{3 \nu}-g_{3 \mu} g_{0 \nu}\right)
$$

In the latter case, the contravariant components of $\beta$ read:

$$
\beta^{\mu}=\frac{1}{T_{0}}(1+a z, 0,0, a t)
$$

thus the unit vector $\hat{\beta}$ is the velocity field of a fluid with constant comoving acceleration along the field lines (for the field line going through $z=0$, the comoving acceleration is $a$ ).

\section{MEAN VALUES OF LOCAL OPERATORS}

Suppose we want to calculate the mean value of a local operator $\widehat{O}(x)$ (in the Heisenberg picture) with the density operator (6):

$$
O(x) \equiv\langle\widehat{O}(x)\rangle=\operatorname{tr}(\widehat{\rho} \widehat{O}(x))_{\text {ren }}=\frac{1}{Z} \operatorname{tr}\left(\exp \left[-\int_{\Sigma} \mathrm{d} \Sigma_{\mu}\left(\widehat{T}^{\mu \nu} \beta_{\nu}-\widehat{\zeta j}^{\mu}\right)\right] \widehat{O}(x)\right)_{\text {ren }}
$$

If $\beta$ is a general field, there is no compelling reason why, at a given point $x$, the mean value $O(x)$ should be simply equal to the same value at the homogeneous global thermodynamic equilibrium with an uniform four-temperature $\beta$ equal to its value in the point $x$, that is $\beta(x)$. For instance, the stress-energy tensor in the point $x$ does not need to be of the ideal form $\sqrt{2}$ with $u=\hat{\beta}(x)$ and $\rho=\rho\left(\beta^{2}, \zeta\right) p=p\left(\beta^{2}, \zeta\right)$ if $\beta$ is not constant. In fact, its tensor structure in (2) is determined by the symmetries of the density operator (1), which is obtained from (6) provided that $\beta$ is constant. 
Nevertheless, one can imagine that if $\beta$ and $\zeta$ are sufficiently slowly varying in space and time, $O(x)$ will be mostly determined by the values of the fields $\beta$ and $\zeta$ around the point $x[9]$. More specifically, the distance over which the thermodynamic fields like $\beta$ vary should be much larger than the typical thermal correlation length, which is governed by the microscopic parameters of the theory and the temperature itself. This can be shown by recasting the fields in the integrand of the eq. (11) as $\beta=\beta(x)+\delta \beta$ and $\zeta=\zeta(x)+\delta \zeta$, so as to obtain:

$$
\begin{aligned}
O(x) & =\frac{1}{Z} \operatorname{tr}\left(\exp \left[-\beta_{\nu}(x) \int_{\Sigma} \mathrm{d} \Sigma_{\mu} \widehat{T}^{\mu \nu}+\zeta(x) \int_{\Sigma} \mathrm{d} \Sigma_{\mu} \widehat{j}^{\mu}-\int_{\Sigma} \mathrm{d} \Sigma_{\mu}\left(\widehat{T}^{\mu \nu} \delta \beta_{\nu}-\delta \zeta \widehat{j}^{\mu}\right)\right] \widehat{O}(x)\right) \widehat{r e n}_{\text {ren }} \\
& =\frac{1}{Z} \operatorname{tr}\left(\exp \left[-\beta_{\nu}(x) \widehat{P}^{\nu}+\zeta(x) \widehat{Q}-\int_{\Sigma} \mathrm{d} \Sigma_{\mu}\left(\widehat{T}^{\mu \nu} \delta \beta_{\nu}-\delta \zeta \widehat{j}^{\mu}\right)\right] \widehat{O}(x)\right)_{\text {ren }}
\end{aligned}
$$

Hence, applying the linear response theory to the exponent above:

$$
\begin{aligned}
O(x) \simeq & \widehat{O}(x)\rangle_{\beta(x)}-\int_{0}^{1} \mathrm{~d} z \int_{\Sigma} \mathrm{d} \Sigma_{\mu}(y)\left(\left\langle\widehat{O}(x) \widehat{T}^{\mu \nu}(y+i z \beta(x))\right\rangle_{\beta(x)}-\langle\widehat{O}(x)\rangle_{\beta(x)}\left\langle\widehat{T}^{\mu \nu}(y+i z \beta(x))\right\rangle_{\beta(x)}\right) \delta \beta_{\nu} \\
& +\int_{0}^{1} \mathrm{~d} z \int_{\Sigma} \mathrm{d} \Sigma_{\mu}(y)\left(\left\langle\widehat{O}(x) \widehat{j}^{\mu}(y+i z \beta(x))\right\rangle_{\beta(x)}-\langle\widehat{O}(x)\rangle_{\beta(x)}\left\langle\widehat{j}^{\mu}(y+i z \beta(x))\right\rangle_{\beta(x)}\right) \delta \zeta
\end{aligned}
$$

where $x$ and $y$ both lie on the hypersurface $\Sigma$. The symbol \langle\rangle$_{\beta}$ stands for the (renormalized) mean value calculated with the homogeneous equilibrium density operator in eq. (1). Particularly, the \langle\rangle$_{\beta(x)}$ stands for the mean value calculated with a fixed four-temperature (and $\zeta$ ) equal to the value of the $\beta$ (and $\zeta$ ) fields in the point $x$. The formula 12 just expresses the aforementioned concept, namely that the local equilibrium value of the operator $\widehat{O}(x)$ is determined by the local values of the thermodynamic fields with corrections depending on quantum-statistical correlations between operators in different points. These correlations - hence the integrand function in eq. 12 - are significant over microscopic distances $l$ dictated by the mass, temperature and coupling constants of the theory, which are supposedly much smaller than the macroscopic distance $L$ over which $\delta \beta$ and $\delta \zeta$ appreciably vary. This condition is usually referred to as hydrodynamical regime and in this regime terms beyond the linear in $\delta \beta$ and $\delta \zeta$ in eq. 12 contribute less and less as they are expected to be suppressed with higher powers of $l / L$.

Under these circumstances, it is possible to expand the thermodynamic fields in the eq. (11) into a Taylor series about the point $x$. For a general $\beta$ field, this method makes it possible to find an approximate expression of the local thermodynamic equilibrium operator $(6)$ as a function of $\beta(x)$ and its derivatives 9 . For the special case of global equilibrium, with constant $\zeta$ and $\beta$ a Killing vector field (7), one can recast the operator $(9)$ so as to have in the exponent the value of the four-temperature in the point $x$ :

$$
\begin{aligned}
O(x) & =\frac{1}{Z} \operatorname{tr}\left(\exp \left[-b_{\mu} \widehat{P}^{\mu}+\frac{1}{2} \varpi_{\mu \nu} \widehat{J}^{\mu \nu}+\zeta \widehat{Q}\right] \widehat{O}(x)\right)_{\text {ren }}=\frac{1}{Z} \operatorname{tr}\left(\exp \left[-\left(b_{\mu}+\varpi_{\mu \nu} x^{\nu}\right) \widehat{P}^{\mu}+\frac{1}{2} \varpi_{\mu \nu} \widehat{J}_{x}^{\mu \nu}+\zeta \widehat{Q}\right] \widehat{O}(x)\right) \\
& =\frac{1}{Z} \operatorname{tr}\left(\exp \left[-\beta_{\mu}(x) \widehat{P}^{\mu}+\frac{1}{2} \varpi_{\mu \nu} \widehat{J}_{x}^{\mu \nu}+\zeta \widehat{Q}\right] \widehat{O}(x)\right)_{\text {ren }}
\end{aligned}
$$

where we have used the angular momentum operators around the point $x$ :

$$
\widehat{J}_{x}^{\mu \nu}=\widehat{J}^{\mu \nu}-x^{\mu} \widehat{P}^{\nu}+x^{\nu} \widehat{P}^{\mu}=\widehat{\mathrm{T}}(x) \widehat{J}^{\mu \nu} \widehat{\mathrm{T}}(x)^{-1}
$$

$\widehat{\mathrm{T}}(x)=\exp [i x \cdot \widehat{P}]$ being the translation operator.

The calculation of mean values 13 is the main purpose of this paper, and, specifically, when $\widehat{O}=\widehat{T}^{\mu \nu}$. We will consider the term in $\varpi$ as small compared with the terms involving $\beta$ and $\zeta$ and expand accordingly. Thus, the leading term in the above equation will be simply the homogeneous equilibrium one with four-temperature equal to its value in the $x$ point, that is the expression 2 with $u=\hat{\beta}(x)$. We will see in the sect. IV that the lowest order corrections to the ideal form are of the second order in $\varpi$ and that they are of either quantum or quantum-relativistic nature as they vanish for $\hbar \rightarrow 0$ or $\hbar / c \rightarrow 0$.

Note that $\beta(x)$ is required to be a future-oriented timelike vector in order to get a finite value for most observables at the lowest order of the $\beta$ expansion. This condition cannot be fulfilled everywhere for the expression (7) if $\varpi \neq 0$. For instance, for the rotating global equilibrium $(3)$, it is easy to check that:

$$
\beta=\frac{1}{T_{0}}(1, \omega \hat{\mathbf{k}} \times \mathbf{x})
$$

which becomes spacelike when $\|\omega \hat{\mathbf{k}} \times \mathbf{x}\|>1$, that is when the velocity exceeds the speed of light. Similarly, for the operator (4), the $\beta$ field $(10)$ is future-oriented timelike only in the Rindler wedge defined by the light cone of the point $(0,0,0,-a / T)$. Therefore, the validity of our calculations will be limited to the physical regions where the $\beta$ field is timelike and with positive time component even though the operator (9) written with the constants $b$ and $\varpi$ does not make this limitation apparent. 


\section{PERTURBATIVE EXPANSION FOR THE STRESS-ENERGY TENSOR}

The goal of this section is to provide an expansion in $\varpi$ for the mean value of the stress-energy tensor in the general form of thermodynamic equilibrium:

$$
T^{\mu \nu}(x)=\frac{1}{Z} \operatorname{tr}\left(\exp \left[-\beta_{\mu}(x) \widehat{P}^{\mu}+\frac{1}{2} \varpi_{\mu \nu} \widehat{J}_{x}^{\mu \nu}+\zeta \widehat{Q}\right] \widehat{T}^{\mu \nu}(x)\right)_{\text {ren }}
$$

Indeed, $\varpi$ is an adimensional tensor in natural units and it the has, in general, very small components. To understand its physical meaning, it is very useful to decompose it into two spacelike vector fields, each having three independent components, projecting along a timelike vector. A physically interesting choice is $u=\hat{\beta}=\beta / \sqrt{\beta^{2}}$, in the regions where $\beta$ given by eq. (7) is timelike. We can then decompose $\varpi$ as follows:

$$
\varpi^{\mu \nu}=\epsilon^{\mu \nu \rho \sigma} w_{\rho} u_{\sigma}+\alpha^{\mu} u^{\nu}-\alpha^{\nu} u^{\mu}
$$

where, by definition:

$$
\alpha^{\mu}(x)=\varpi^{\mu \nu} u_{\nu} \quad w^{\mu}(x)=-\frac{1}{2} \epsilon^{\mu \nu \rho \sigma} \varpi_{\nu \rho} u_{\sigma}
$$

Note that $\alpha$ and $w$, unlike $\varpi$, are not constant and they are both orthogonal to $u$, hence spacelike. The physical meaning of $\alpha$ and $w$ vectors can be shown starting from the eq. (5). Because of (7) and (5) at equilibrium one has:

$$
\varpi_{\mu \nu}=\partial_{\nu} \beta_{\mu}
$$

whence:

$$
\alpha^{\mu}=\varpi^{\mu \nu} u_{\nu}=u_{\nu} \partial^{\nu} \beta^{\mu}=u^{\mu} u_{\nu} \partial^{\nu} \sqrt{\beta^{2}}+\sqrt{\beta^{2}} u_{\nu} \partial^{\nu} u^{\mu}
$$

We can now take the scalar product with $u^{\mu}$ and conclude that:

$$
u_{\nu} \partial^{\nu} \sqrt{\beta^{2}} \equiv D \sqrt{\beta^{2}}=0
$$

which tells us that, as expected, at the thermodynamic equilibrium the comoving temperature along the flow lines does not change and $\partial_{\mu} \beta^{2}=\nabla_{\mu} \beta^{2}$, where:

$$
\nabla_{\mu} \equiv \partial_{\mu}-u_{\mu} D
$$

Thereby, the $\alpha$ vector simply becomes:

$$
\alpha^{\mu}=\sqrt{\beta^{2}} u_{\nu} \partial^{\nu} u^{\mu}=\sqrt{\beta^{2}} D u^{\mu}=\frac{1}{T} a^{\mu}
$$

that is the acceleration field divided by the proper temperature. Note also that, being $\partial_{\mu} \beta_{\nu}+\partial_{\nu} \beta_{\mu}=0$, one has:

$$
0=u^{\nu}\left(\partial_{\nu} \beta_{\mu}+\partial_{\mu} \beta_{\nu}\right)=\alpha_{\mu}+\frac{1}{2 \sqrt{\beta^{2}}} \partial_{\mu} \beta^{2}=\frac{1}{T} a_{\mu}-\frac{1}{T^{2}} \nabla_{\mu} T
$$

Likewise, it can be shown that $w$ corresponds to an angular velocity divided by a temperature, for, by using (8)

$$
w^{\mu}=-\frac{1}{2} \epsilon^{\mu \nu \rho \sigma} \varpi_{\nu \rho} u_{\sigma}=\frac{1}{2} \epsilon^{\mu \nu \rho \sigma}\left(\partial_{\nu} \beta_{\rho}\right) u_{\sigma}=\frac{1}{2} \epsilon^{\mu \nu \rho \sigma} \sqrt{\beta^{2}} u_{\sigma} \partial_{\nu} u_{\rho}=\frac{1}{2 T} \epsilon^{\mu \nu \rho \sigma} u_{\sigma} \nabla_{\nu} u_{\rho}=\frac{1}{T} \omega^{\mu}
$$

being $\omega^{\mu}=\frac{1}{2} \epsilon^{\mu \nu \rho \sigma} u_{\sigma} \nabla_{\nu} u_{\rho}$, as it is known in literature, the local vorticity vector. Restoring the physical constants, one then has the adimensional four-vectors:

$$
\alpha_{\mu}=\frac{\hbar a_{\mu}}{c K T} \quad w_{\mu}=\frac{\hbar \omega_{\mu}}{K T}
$$

These numbers are, for the vast majority of physical systems, much less than 1 and a perturbative expansion in $\varpi$ of the eq. 15] is then feasible. They can give rise to relevant corrections if the implied additional terms to the ideal 
stress-energy tensor are some sizeable fraction thereof or when these terms are comparable to the viscous tensor. According to the eq. 21), this happens at for very large accelerations or very low temperatures. Hence, let us define:

$$
\widehat{\mathcal{R}}(\varpi) \equiv \exp \left[-\beta_{\mu}(x) \widehat{P}^{\mu}+\frac{1}{2} \varpi_{\mu \nu} \widehat{J}_{x}^{\mu \nu}+\zeta \widehat{Q}\right]=\exp \left[-\beta_{\mu}(x) \widehat{P}^{\mu}+\frac{1}{2} \varpi_{\mu \nu} \widehat{J}_{x}^{\mu \nu}\right] \exp [\zeta \widehat{Q}]
$$

where, in the last equality, advantage has been taken of the supposed commutation of the charge operator $\widehat{Q}$ with both the $\widehat{P}$ 's and $\widehat{J}$ 's. At the second order in $\varpi$ one can write:

$$
\widehat{\mathcal{R}}(\varpi)=\widehat{\mathcal{R}}^{(0)}+\varpi_{\mu \nu} \widehat{\mathcal{R}}^{(1) \mu \nu}+\varpi_{\mu \nu} \varpi_{\rho \sigma} \widehat{\mathcal{R}}^{(2) \mu \nu \rho \sigma}+o\left(\varpi^{2}\right)
$$

and, by using the Poincaré group commutation relations, it can be shown that (see Appendix A):

$$
\begin{aligned}
\widehat{\mathcal{R}}^{(0)} & =\mathrm{e}^{-\beta \cdot \widehat{P}+\zeta \widehat{Q}} \\
\widehat{\mathcal{R}}^{(1) \mu \nu} & =\frac{1}{4}\left\{\mathrm{e}^{-\beta \cdot \widehat{P}+\zeta \widehat{Q}}, \widehat{J}^{\mu \nu}\right\}, \\
\widehat{\mathcal{R}}^{(2) \mu \nu \rho \sigma} & =\frac{1}{16}\left\{\mathrm{e}^{-\beta \cdot \widehat{P}+\zeta \widehat{Q}}, \widehat{J}^{\mu \nu} \widehat{J}^{\rho \sigma}\right\}+\frac{1}{8} \mathrm{e}^{-\beta \cdot \widehat{P}+\zeta \widehat{Q}} \beta^{\mu} \beta^{\rho} \widehat{P}^{\nu} \widehat{P}^{\sigma}-\frac{1}{12} \mathrm{e}^{-\beta \cdot \widehat{P}+\zeta \widehat{Q}} \beta^{\mu} g^{\nu \rho} \widehat{P}^{\sigma} .
\end{aligned}
$$

where the curly bracket expression $\{$,$\} stands for the anticommutator.$

By using the eqs. (23) and (24), the mean value 15 can be expressed as an expansion in $\varpi$ with coefficients which are calculated at the homogeneous thermodynamic equilibrium:

$$
\begin{aligned}
T^{\mu \nu}(x)= & \frac{\operatorname{tr}\left(\widehat{\mathcal{R}}(\varpi) \widehat{T}^{\mu \nu}(x)\right)}{\operatorname{tr}(\widehat{\mathcal{R}}(\varpi))}=\left\langle\widehat{T}^{\mu \nu}(x)\right\rangle_{\beta(x)}+\frac{1}{2} \varpi_{\rho \sigma} \operatorname{Re}\left\langle\widehat{J}_{x}^{\rho \sigma} ; \widehat{T}^{\mu \nu}(x)\right\rangle_{\beta(x)} \\
& +\varpi_{\rho \sigma} \varpi_{\lambda \tau}\left[\frac{1}{8} \operatorname{Re}\left\langle\widehat{J}_{x}^{\rho \sigma} \widehat{J}_{x}^{\lambda \tau} ; \widehat{T}^{\mu \nu}(x)\right\rangle_{\beta(x)}+\frac{1}{8} \beta^{\rho}(x) \beta^{\lambda}(x)\left\langle\widehat{P}^{\sigma} \widehat{P}^{\tau} ; \widehat{T}^{\mu \nu}(x)\right\rangle_{\beta(x)}-\frac{1}{12} \beta^{\rho}(x) g^{\lambda \sigma}\left\langle\widehat{P}^{\tau} ; \widehat{T}^{\mu \nu}(x)\right\rangle_{\beta(x)}\right. \\
& \left.-\frac{1}{4} \operatorname{Re}\left\langle\widehat{J}_{x}^{\rho \sigma} ; \widehat{T}^{\mu \nu}(x)\right\rangle_{\beta(x)}\left\langle\widehat{J}_{x}^{\lambda \tau}\right\rangle_{\beta(x)}\right]+o\left(\varpi^{2}\right)
\end{aligned}
$$

where we have used the relations for two hermitian operators $\widehat{A}, \widehat{B}$ :

$$
\operatorname{Re}\langle\widehat{A} \widehat{B}\rangle=\frac{1}{2}\langle\{\widehat{A}, \widehat{B}\}\rangle \quad i \operatorname{Im}\langle\widehat{A} \widehat{B}\rangle=\frac{1}{2}\langle[\widehat{A}, \widehat{B}]\rangle
$$

and the notation has been introduced:

$$
\langle\widehat{A} ; \widehat{B}\rangle=\langle\widehat{A} \widehat{B}\rangle-\langle\widehat{A}\rangle\langle\widehat{B}\rangle
$$

for the correlator between $\widehat{A}$ and $\widehat{B}$. The terms in eq. 25. containing $\widehat{P}$ can be readily calculated taking the derivative of $\langle\widehat{T}\rangle_{\beta}$ with respect to $\beta$. Indeed:

$$
\begin{aligned}
\beta^{\rho} g^{\lambda \sigma} \frac{\partial}{\partial \beta_{\tau}}\left\langle\widehat{T}^{\mu \nu}\right\rangle_{\beta} & =-\beta^{\rho} g^{\lambda \sigma}\left\langle\widehat{P}^{\tau} ; \widehat{T}^{\mu \nu}\right\rangle_{\beta} \\
\beta^{\rho} \beta^{\lambda} \frac{\partial^{2}}{\partial \beta_{\sigma} \partial \beta_{\tau}}\left\langle\widehat{T}^{\mu \nu}\right\rangle_{\beta} & =\beta^{\rho} \beta^{\lambda}\left(\left\langle\widehat{P}^{\sigma} \widehat{P}^{\tau} ; \widehat{T}^{\mu \nu}\right\rangle_{\beta}-\left\langle\widehat{P}^{\sigma} ; \widehat{T}^{\mu \nu}\right\rangle_{\beta}\left\langle\widehat{P}^{\tau}\right\rangle_{\beta}-\left\langle\widehat{P}^{\tau} ; \widehat{T}^{\mu \nu}\right\rangle_{\beta}\left\langle\widehat{P}^{\sigma}\right\rangle_{\beta}\right)
\end{aligned}
$$

Note that the two rightmost terms in the last equation vanish once multiplied by $\varpi_{\rho \sigma} \varpi_{\lambda \tau}$ for, being $\langle\widehat{P}\rangle_{\beta} \propto \beta$, they contain the symmetric combination $\beta^{\lambda} \beta^{\tau}$ or $\beta^{\rho} \beta^{\sigma}$.

All mean values in eq. 25) involving angular momentum operators can be rewritten in a form which makes it apparent that their dependence on $x$ is only through the value of the four-temperature, by taking advantage of the translational invariance of the density operator. For instance:

$$
\left\langle\widehat{J}_{x}^{\rho \sigma} \widehat{J}_{x}^{\lambda \tau} ; \widehat{T}^{\mu \nu}(x)\right\rangle_{\beta(x)}=\left\langle\widehat{\mathrm{T}}^{-1}(x) \widehat{J}_{x}^{\rho \sigma} \widehat{J}_{x}^{\lambda \tau} \widehat{\mathrm{T}}(x) ; \widehat{\mathrm{T}}^{-1}(x) \widehat{T}^{\mu \nu}(x) \widehat{\mathrm{T}}(x)\right\rangle_{\beta(x)}=\left\langle\widehat{J}^{\rho \sigma} \widehat{J}^{\lambda \tau} ; \widehat{T}^{\mu \nu}(0)\right\rangle_{\beta(x)}
$$

and similarly for the others, where eq. 14 has been used. Then, it is convenient to decompose the tensor $\widehat{J}$ into two spacelike vector operators the same fashion as for $\varpi$ in eq. 16):

$$
\widehat{J}^{\mu \nu}=u^{\mu} \widehat{K}^{\nu}-\widehat{K}^{\mu} u^{\nu}+\epsilon^{\mu \nu \rho \sigma} \widehat{J}_{\rho} u_{\sigma}
$$


being

$$
\widehat{K}^{\mu}=u_{\rho} \widehat{J}^{\rho \mu} \quad \widehat{J}^{\mu}=-\frac{1}{2} \epsilon^{\mu \rho \sigma \tau} \widehat{J}_{\rho \sigma} u_{\tau}
$$

The operators $\widehat{J}^{\mu}$ and $\widehat{K}^{\mu}$ are simply the generators of the rotation and boosts with respect to the reference frame with time direction $u$. Using the invariance by rotation (in the hyperplane orthogonal to $u$ ), parity and time reversal, which are assumed to hold for our hamiltonian, one readily obtains that (see Appendix B):

$$
\operatorname{Re}\left\langle\widehat{J}^{\rho \sigma} ; \widehat{T}^{\mu \nu}(0)\right\rangle_{\beta(x)}=0 \quad\left\langle\widehat{J}^{\rho \sigma}\right\rangle_{\beta(x)}=0
$$

Therefore, plugging the decomposition (27) into the eq. $(25)$, and using the relations $(17)$ and $(26)$ and after the removal of the vanishing terms, the eq. (25) can be written as:

$$
\begin{aligned}
T^{\mu \nu}(x) & =\left\langle\widehat{T}^{\mu \nu}(x)\right\rangle_{\beta(x)}+\frac{1}{2} \alpha_{\rho} \alpha_{\sigma} \operatorname{Re}\left\langle\widehat{K}^{\rho} \widehat{K}^{\sigma} ; \widehat{T}^{\mu \nu}(0)\right\rangle_{\beta(x)}+\frac{1}{2} w_{\rho} w_{\sigma} \operatorname{Re}\left\langle\widehat{J}^{\rho} \widehat{J}^{\sigma} ; \widehat{T}^{\mu \nu}(0)\right\rangle_{\beta(x)} \\
& +\frac{1}{2} \alpha_{\rho} w_{\sigma} \operatorname{Re}\left\langle\left\{\widehat{J}^{\rho}, \widehat{K}^{\sigma}\right\} ; \widehat{T}^{\mu \nu}(0)\right\rangle_{\beta(x)}+\frac{1}{8} \beta^{\rho} \beta^{\lambda} \frac{\partial^{2}}{\partial \beta_{\sigma} \partial \beta_{\tau}}\left\langle\widehat{T}^{\mu \nu}(x)\right\rangle_{\beta(x)}+\frac{1}{12} \beta^{\rho} g^{\lambda \sigma} \frac{\partial}{\partial \beta_{\tau}}\left\langle\widehat{T}^{\mu \nu}(x)\right\rangle_{\beta(x)}+o\left(\varpi^{2} \gamma 28\right)
\end{aligned}
$$

The derivative terms are easy to work out by using (2); they will give rise to expressions involving the thermodynamic functions pressure, energy density and their derivatives, that is specific heats. On the other hand, the correlators in eq. 28) cannot be expressed in terms of known thermodynamic functions. In fact, they can be written as linear combinations of new thermodynamic coefficients which can be expressed in turn as correlators of specific components of the stress-energy tensor and angular momentum or boost operators $\widehat{J}$ and $\widehat{K}$, that is

$$
\begin{aligned}
& \frac{1}{2} \operatorname{Re}\left\langle\left\{\widehat{K}^{\rho}, \widehat{K}^{\sigma}\right\} ; \widehat{T}^{\mu \nu}(0)\right\rangle_{\beta(x)}=-u^{\mu} u^{\nu} \Delta^{\rho \sigma} k_{t}(T, \zeta)+\Delta^{\mu \nu} \Delta^{\rho \sigma} k_{\theta}(T, \zeta)+\left(\Delta^{\mu \sigma} \Delta^{\rho \nu}+\Delta^{\nu \sigma} \Delta^{\rho \mu}-\frac{2}{3} \Delta^{\mu \nu} \Delta^{\rho \sigma}\right) k_{s}(T, \zeta) \\
& \frac{1}{2} \operatorname{Re}\left\langle\left\{\widehat{J}^{\rho}, \widehat{J}^{\sigma}\right\} ; \widehat{T}^{\mu \nu}(0)\right\rangle_{\beta(x)}=-u^{\mu} u^{\nu} \Delta^{\rho \sigma} j_{t}(T, \zeta)+\Delta^{\mu \nu} \Delta^{\rho \sigma} j_{\theta}(T, \zeta)+\left(\Delta^{\mu \sigma} \Delta^{\rho \nu}+\Delta^{\nu \sigma} \Delta^{\rho \mu}-\frac{2}{3} \Delta^{\mu \nu} \Delta^{\rho \sigma}\right) j_{s}(T, \zeta) \\
& \operatorname{Re}\left\langle\left\{\widehat{K}^{\rho}, \widehat{J}^{\sigma}\right\} ; \widehat{T}^{\mu \nu}(0)\right\rangle_{\beta(x)}=\left(u_{\mu} u_{\kappa} \epsilon^{\kappa \rho \sigma \nu}+u^{\nu} u_{\kappa} \epsilon^{\kappa \rho \sigma \mu}\right) l_{v}(T, \zeta)
\end{aligned}
$$

where

$$
\Delta^{\mu \nu} \equiv g^{\mu \nu}-u^{\mu} u^{\nu}
$$

is the projector onto the hyperplane orthogonal to $u$ and

$$
\begin{array}{llrl}
k_{t}(T, \zeta) & =\operatorname{Re}\left\langle\widehat{K}^{3} \widehat{K}^{3} ; \widehat{T}^{00}(0)\right\rangle_{T} & k_{\theta}(T, \zeta)=\frac{1}{3} \operatorname{Re} \sum_{i=1}^{3}\left\langle\widehat{K}^{3} \widehat{K}^{3} ; \widehat{T}^{i i}(0)\right\rangle_{T} & k_{s}(T, \zeta)=\operatorname{Re}\left\langle\widehat{K}^{1} \widehat{K}^{2} ; \widehat{T}^{12}(0)\right\rangle_{T} \\
j_{t}(T, \zeta)=\operatorname{Re}\left\langle\widehat{J}^{3} \widehat{J}^{3} ; \widehat{T}^{00}(0)\right\rangle_{T} & j_{\theta}(T, \zeta)=\frac{1}{3} \operatorname{Re} \sum_{i=1}^{3}\left\langle\widehat{J}^{3} \widehat{J}^{3} ; \widehat{T}^{i i}(0)\right\rangle_{T} & j_{s}(T, \zeta)=\operatorname{Re}\left\langle\widehat{J}^{1} \widehat{J}^{2} ; \widehat{T}^{12}(0)\right\rangle_{T} \\
l_{v}(T, \zeta)=\operatorname{Re}\left\langle\left\{\widehat{K}^{1}, \widehat{J}^{2}\right\} ; \widehat{T}^{03}(0)\right\rangle_{T} & &
\end{array}
$$

In the eq. 30 the notation \langle\rangle$_{T}$ has been introduced meaning that the expectation value is calculated in the rest frame where $\beta=(1 / T, \mathbf{0})$. The derivation of eqs. 29) and (30) can be found in Appendix B.

Finally, after having worked out the derivatives of the stress-energy tensor and using the eqs. (29) and (2) in the eq. 28, , one obtains:

$$
T^{\mu \nu}(x)=\left(\rho-\alpha^{2} U_{\alpha}-w^{2} U_{w}\right) u^{\mu} u^{\nu}-\left(p-\alpha^{2} D_{\alpha}-w^{2} D_{w}\right) \Delta^{\mu \nu}+A \alpha^{\mu} \alpha^{\nu}+W w^{\mu} w^{\nu}+G\left(u^{\mu} \gamma^{\nu}+\gamma^{\mu} u^{\nu}\right)+o\left(\varpi^{2}\right)
$$

where $\rho, p$ are the usual homogeneous thermodynamic equilibrium functions energy density and pressure, and the functions $U, D, A, W, G$ read:

$$
\begin{aligned}
& U_{\alpha}=\frac{1}{24} T \frac{\partial \rho}{\partial T}+\frac{1}{4}(\rho+p)+\frac{1}{2} k_{t} \\
& U_{w}=\frac{1}{2} j_{t} \\
& D_{w}=\frac{1}{2} j_{\theta}-\frac{1}{3} j_{s} \\
& A=\frac{1}{4}(\rho+p)+k_{s} \quad W=j_{s} \\
& G=\frac{1}{2} l_{v}-\frac{1}{12}(\rho+p) \\
& D_{\alpha}=\frac{1}{24}(\rho+p)+\frac{1}{2} k_{\theta}-\frac{1}{3} k_{s}
\end{aligned}
$$


The vector $\gamma^{\mu}$ in eq. 31 is defined as:

$$
\gamma^{\mu}=(\alpha \cdot \varpi)_{\lambda} \Delta^{\lambda \mu}=\epsilon^{\mu \nu \rho \sigma} w_{\nu} \alpha_{\rho} u_{\sigma}
$$

where the $\varpi$ decomposition 16 has been used,

As it can be seen from eq. (31), the stress-energy tensor has corrections to its ideal form which depend on quadratic combinations of the two vector fields, $\alpha$ and $w$ arising from the decomposition of the exterior derivative of the temperature four-vector $\beta$. At thermodynamic equilibrium, according to the previous discussion and the eqs. 21, they are proportional to the acceleration $a^{\mu}$ and angular velocity (or vorticity) $\omega^{\mu}$, so that the eq. (31) can be rewritten in the most suggestive fashion by restoring the natural constants as:

$$
\begin{aligned}
T^{\mu \nu}(x)= & {\left[\rho+\left(\frac{\hbar|a|}{c K T}\right)^{2} U_{\alpha}+\left(\frac{\hbar|\omega|}{K T}\right)^{2} U_{w}\right] u^{\mu} u^{\nu}-\left[p+\left(\frac{\hbar|a|}{c K T}\right)^{2} D_{\alpha}+\left(\frac{\hbar|\omega|}{K T}\right)^{2} D_{w}\right] \Delta^{\mu \nu} } \\
& +A\left(\frac{\hbar|a|}{c K T}\right)^{2} \hat{a}^{\mu} \hat{a}^{\nu}+W\left(\frac{\hbar|\omega|}{K T}\right)^{2} \hat{\omega}^{\mu} \hat{\omega}^{\nu}+G \frac{\hbar^{2}|\omega||a|}{c(K T)^{2}}\left(u^{\mu} \hat{\gamma}^{\nu}+\hat{\gamma}^{\mu} u^{\nu}\right)+o\left(\varpi^{2}\right)
\end{aligned}
$$

where $|a|=\sqrt{-a_{\mu} a^{\mu}}$ and $|\omega|=\sqrt{-\omega_{\mu} \omega^{\mu}}$ and $\hat{a}, \hat{\omega}$ are the corresponding unit vectors. In the expression (34) the adimensional scales $\hbar a / c K T$ and $\hbar \omega / K T$ involving acceleration and vorticity have been separated from the thermodynamic functions $U, A, W, D, G$ having the same dimension as $\rho$ and/or $p$ making it easier to appreciate the size of the correction to the ideal form.

\section{A. Relation with other second-order hydrodynamical coefficient calculations}

The appearance of extra terms in the stress-energy tensor at thermodynamic equilibrium with respect to its ideal form has, needless to say, several physical consequences. The presence of non-dissipative quadratic corrections in the vorticity and gradients of temperature (hence accelerations at equilibrium, according to eq. (19) was pointed out in ref. [3, 4] and, being non dissipative in nature, defined as thermodynamic in ref. [6]. The calculation of such coefficients has attracted much attention lately (see 12 for a recent review), especially in conformal field theories [13 15] with different techniques [16, 17] (see also ref. [18]). The coefficients that we have denoted by $D_{\alpha}, D_{w}, A$ and $W$ are in the following relation with those known as $\xi_{3}, \xi_{4}, \lambda_{3}, \lambda_{4}$ in literature:

$$
\begin{array}{lll}
\frac{A}{T^{2}}=9 \lambda_{4} & \frac{W}{T^{2}}=\lambda_{3} \\
\frac{D_{w}}{T^{2}}=\left(\frac{\lambda_{3}}{3}-2 \xi_{3}\right) & \frac{D_{\alpha}}{T^{2}}=\left(3 \lambda_{4}-9 \xi_{4}\right)
\end{array}
$$

Remarkably, the number of coefficients quoted in (34) is larger than envisaged in ref. [3, 4, and the reason is that we did not assume, as it is usually done in the Landau frame, that the proper energy density $\rho$ has the same functional dependence on the temperature as at homogeneous thermodynamic equilibrium. This assumption proves to be incorrect, and the extra coefficients cannot be reabsorbed by a redefinition of temperature, as it will be discussed and shown in sect. VI

Before tackling these issues, it is necessary to calculate the coefficients $U, D, A, W, G$ in some instance and we will do it for the simplest case of a real scalar free field. As it will be clear from the calculations shown in the next section, they all have a classical expression in the massive case, and, as a consequence, all the corrections in eq. (34) to the ideal form turn out to be of quantum origin, as they vanish in the $\hbar \rightarrow 0$ limit.

\section{THE FREE SCALAR FIELD}

The goal of this section is to calculate the coefficients in eq. 34 for a free real scalar field. This implies $\zeta=0$ in the density operator (1), yet it is quite easy to extend the obtained results to the charged case with $\zeta \neq 0$ in the Boltzmann limit of distinguishable particles.

The theory is described by the Lagrangian density:

$$
\mathcal{L}=\frac{1}{2} \partial_{\mu} \widehat{\psi} \partial^{\mu} \widehat{\psi}-\frac{1}{2} m^{2} \widehat{\psi}^{2} .
$$


By adding the super-potential

$$
-2 \xi \partial_{\mu}\left(\widehat{\psi} \partial^{\mu} \widehat{\psi}\right)
$$

a class of stress-energy tensors can be obtained as Noether currents associated to space-time translations. Although they are explicitely dependent on the parameter $\xi$, they differ from each other by a divergence:

$$
\widehat{T}_{\xi}^{\mu \nu}=\partial^{\mu} \widehat{\psi} \partial^{\nu} \widehat{\psi}-\frac{1}{2} g^{\mu \nu}\left(\partial_{\lambda} \widehat{\psi} \partial^{\lambda} \widehat{\psi}-m^{2} \widehat{\psi}^{2}\right)+2 \xi \partial_{\lambda}\left(g^{\mu \nu} \widehat{\psi} \partial^{\lambda} \widehat{\psi}-g^{\lambda \mu} \widehat{\psi} \partial^{\nu} \widehat{\psi}\right)
$$

thus they lead to the same generators of the Poincaré group. For $\xi=0$ the tensor is the so-called canonical stressenergy tensor, while for $\xi=1 / 6$ the tensor is the so-called improved stress-energy tensor [19. In the translationally invariant homogeneous equilibrium (1) all mean values of local operators are independent of $x$, thus the divergence in the above expression vanishes, hence $\rho$ and $p$ do not depend on $\xi$. In fact, as we will show, this is not true in the case of generalized equilibrium and the correlators in eq. (30) are explicitely dependent on $\xi$.

At the very beginning, it should be pointed out that in principle one should use normal ordering in the calculation of the mean values of $\widehat{T}$ in a free field theory to subtract zero point infinity. However, this is not needed in the calculation of a correlator such as $\left\langle\widehat{J}^{\mu \nu} \widehat{J}^{\rho \sigma} ; \widehat{T}^{\alpha \beta}(0)\right\rangle_{T}$ because $: \widehat{T}:=\widehat{T}-\langle 0|\widehat{T}| 0\rangle(\widehat{T}$ being a quadratic operator in the fields) so that the vacuum term cancels out in the subtraction $\left\langle\widehat{J}^{\mu \nu} \widehat{J}^{\rho \sigma} \widehat{T}^{\alpha \beta}(0)\right\rangle_{T}-\left\langle\widehat{J}^{\mu \nu} \widehat{J}^{\rho \sigma}\right\rangle_{T}\left\langle\widehat{T}^{\alpha \beta}(0)\right\rangle_{T}$.

The basic tool we need in order to carry out the calculation is the free field $n$-points Wightman thermal function:

$$
\mathcal{W}_{T}^{(n)}\left(x_{1}, x_{2}, \ldots, x_{n}\right)=\left\langle\widehat{\psi}\left(x_{1}\right) \widehat{\psi}\left(x_{2}\right) \ldots \widehat{\psi}\left(x_{n}\right)\right\rangle_{T}
$$

which can be written in terms of 2-points thermal functions according to a version of the Wick theorem [20] suitable for thermal field theory. For an even $n$ :

$$
\mathcal{W}_{T}^{(n)}\left(x_{1}, \ldots, x_{n}\right)=\sum_{j=2}^{n}\left[\mathcal{W}_{T}^{(2)}\left(x_{1}, x_{j}\right) \mathcal{W}_{T}^{(n-2)}\left(x_{2}, \ldots, x_{j-1}, x_{j+1}, \ldots, x_{n}\right)\right],
$$

while, if $n$ is odd, $\mathcal{W}_{T}^{(n)}\left(x_{1}, \ldots, x_{n}\right)=0$. In the case of a free real scalar field, the 2-points Wightman thermal function reads:

$$
\mathcal{W}_{T}^{(2)}(x, y)=\frac{1}{(2 \pi)^{3}} \int \mathrm{d}^{4} k e^{-i k(x-y)}\left[\theta\left(k^{0}\right)+n_{T}\left(\left|k^{0}\right|\right)\right] \delta\left(k^{2}-m^{2}\right)
$$

where

$$
n_{T}(\varepsilon)=\frac{1}{\mathrm{e}^{\varepsilon / T}-1}
$$

is the Bose-Einstein distribution. We then define

$$
\mathcal{T}_{T}^{\mu \nu|\rho \sigma| \alpha \beta}(x, y, z)=\left\langle\widehat{T}^{\mu \nu}(x) \widehat{T}^{\rho \sigma}(y) \widehat{T}^{\alpha \beta}(z)\right\rangle_{T}-\left\langle\widehat{T}^{\mu \nu}(x) \widehat{T}^{\rho \sigma}(y)\right\rangle_{T}\left\langle\widehat{T}^{\alpha \beta}(z)\right\rangle_{T},
$$

which can be calculated with the point-split procedure as

$$
\mathcal{T}_{T}^{\mu \nu|\rho \sigma| \alpha \beta}(x, y, z)=\Theta_{x}^{\mu \nu} \Theta_{y}^{\rho \sigma} \Theta_{z}^{\alpha \beta}\left[\mathcal{W}_{T}^{(6)}\left(x_{1}, x_{2}, y_{1}, y_{2}, z_{1}, z_{2}\right)-\mathcal{W}_{T}^{(4)}\left(x_{1}, x_{2}, y_{1}, y_{2}\right) \mathcal{W}_{T}^{(2)}\left(z_{1}, z_{2}\right)\right]
$$

where

$$
\Theta_{x}^{\mu \nu}=\left\{(1-2 \xi) \partial_{x_{1}}^{\mu} \partial_{x_{2}}^{\nu}-2 \xi \partial_{x_{2}}^{\mu} \partial_{x_{2}}^{\nu}+\frac{1}{2} g^{\mu \nu}\left[(4 \xi-1) \partial_{x_{1}} \cdot \partial_{x_{2}}+4 \xi \square_{x_{2}}+m^{2}\right]\right\}_{x_{1}, x_{2} \rightarrow x} .
$$

The general expression of the correlators is then:

$$
\begin{aligned}
\left\langle\widehat{J}^{\mu \nu} \widehat{J}^{\rho \sigma} ; \widehat{T}^{\alpha \beta}(0)\right\rangle_{T}= & \int \mathrm{d}^{3} \mathrm{xd}^{3} \mathrm{y}\left[x^{\mu} y^{\rho} \mathcal{T}_{T}^{0 \nu|0 \sigma| \alpha \beta}(x, y, 0)-x^{\nu} y^{\rho} \mathcal{T}_{T}^{0 \mu|0 \sigma| \alpha \beta}(x, y, 0)\right. \\
& \left.-x^{\mu} y^{\sigma} \mathcal{T}_{T}^{0 \nu|0 \rho| \alpha \beta}(x, y, 0)+x^{\nu} y^{\sigma} \mathcal{T}_{T}^{0 \mu|0 \rho| \alpha \beta}(x, y, 0)\right]
\end{aligned}
$$

with $x^{0}=y^{0}=0$ because the $\widehat{J}$ 's are time-independent. 


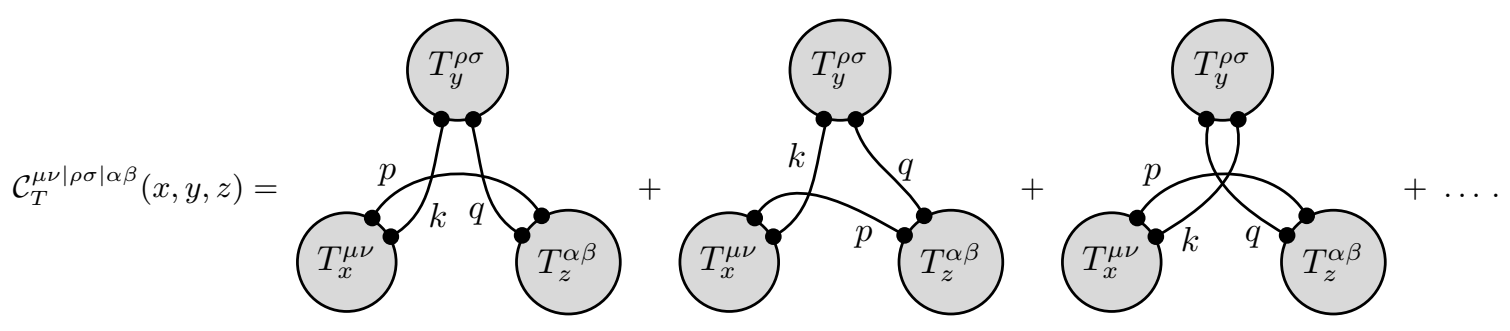

Out of the 15 different diagrams stemming from the contractions of the 6-point Wightman thermal function, in $\mathcal{T}$ some are cancelled by the subtraction term, leaving only the 12 diagrams in which $\widehat{T}^{\alpha \beta}(z)$ is not a disconnected component. Since $\left\langle\widehat{J}^{\mu \nu}\right\rangle_{T}=0$, in the eq. $(37)$ the remaining 4 disconnected graphs in $\mathcal{T}$ do not contribute to the result. Therefore in (37) we can replace $\mathcal{T}$ with its connected subset of 8 diagrams and we get:

$$
\begin{array}{r}
\mathcal{C}_{T}^{\mu \nu|\rho \sigma| \alpha \beta}(x, y, 0)=\frac{1}{(2 \pi)^{9}} \int \mathrm{d}^{4} k \mathrm{~d}^{4} p \mathrm{~d}^{4} q \mathrm{e}^{-i(k+p) x} \mathrm{e}^{-i(q-k) y} \mathcal{P}^{\mu \nu|\rho \sigma| \alpha \beta}(k, p, q) \delta\left(k^{2}-m^{2}\right) \delta\left(p^{2}-m^{2}\right) \delta\left(q^{2}-m^{2}\right) \\
\times\left[\theta\left(k^{0}\right)+n_{T}\left(\left|k^{0}\right|\right)\right]\left[\theta\left(p^{0}\right)+n_{T}\left(\left|p^{0}\right|\right)\right]\left[\theta\left(q^{0}\right)+n_{T}\left(\left|q^{0}\right|\right)\right],
\end{array}
$$

with

$$
\begin{aligned}
\mathcal{P}^{\mu \nu|\rho \sigma| \alpha \beta}(k, p, q)=\{ & \left.-(1-2 \xi)\left(k^{\mu} p^{\nu}+p^{\mu} k^{\nu}\right)+2 \xi\left(k^{\mu} k^{\nu}+p^{\mu} p^{\nu}\right)-g^{\mu \nu}\left[(4 \xi-1) k \cdot p+2 \xi\left(k^{2}+p^{2}\right)-m^{2}\right]\right\} \\
\times & \left\{(1-2 \xi)\left(k^{\rho} q^{\sigma}+q^{\rho} k^{\sigma}\right)+2 \xi\left(k^{\rho} k^{\sigma}+q^{\rho} q^{\sigma}\right)-g^{\rho \sigma}\left[-(4 \xi-1) k \cdot q+2 \xi\left(k^{2}+q^{2}\right)-m^{2}\right]\right\} \\
& \times\left\{-(1-2 \xi)\left(p^{\alpha} q^{\beta}+q^{\alpha} p^{\beta}\right)+2 \xi\left(p^{\alpha} p^{\beta}+q^{\alpha} q^{\beta}\right)-g^{\alpha \beta}\left[(4 \xi-1) p \cdot q+2 \xi\left(p^{2}+q^{2}\right)-m^{2}\right]\right\} .
\end{aligned}
$$

The thermodynamic correlators in eq. (30) can be found by selecting the suitable indices in eq. (37). For instance, for the $k_{t}$ correlator:

$$
k_{t}(T)=\left.\int \mathrm{d}^{3} \mathrm{xd}^{3} \mathrm{y} x^{3} y^{3} \mathcal{C}_{T}^{00|00| 00}(x, y, 0)\right|_{x^{0}=y^{0}=0}
$$

Using

$$
\int \mathrm{d}^{3} \mathrm{xd}^{3} \mathrm{y} x^{i} y^{j} \mathrm{e}^{i(\mathbf{k}+\mathbf{p}) \cdot \mathbf{x}} \mathrm{e}^{i(\mathbf{q}-\mathbf{k}) \cdot \mathbf{y}}=-(2 \pi)^{6} \partial_{p_{i}} \delta(\mathbf{p}-\mathbf{k}) \partial_{q_{j}} \delta(\mathbf{q}-\mathbf{k})
$$

and

$$
\delta\left(k^{2}-m^{2}\right)=\frac{1}{2 \varepsilon_{\mathbf{k}}}\left[\delta\left(k^{0}+\varepsilon_{\mathbf{k}}\right)+\delta\left(k^{0}-\varepsilon_{\mathbf{k}}\right)\right],
$$

where $\varepsilon_{\mathbf{k}}=\sqrt{\mathbf{k}^{2}+m^{2}}$, one can then integrate in $\mathbf{x}$ and $\mathbf{y}$, thereafter in $k^{0}, p^{0}, q^{0}$ so as to obtain

$$
k_{t}(T)=-\frac{1}{(2 \pi)^{3}} \int \mathrm{d}^{3} \mathrm{kd}^{3} \mathrm{pd}^{3} \mathrm{q} \frac{1}{8 \varepsilon_{\mathbf{k}} \varepsilon_{\mathbf{p}} \varepsilon_{\mathbf{q}}}\left(\mathcal{S}_{+++}+\cdots+\mathcal{S}_{---}\right) \partial_{p_{3}} \delta(\mathbf{p}-\mathbf{k}) \partial_{q_{3}} \delta(\mathbf{q}-\mathbf{k}),
$$

where the $\mathcal{S}$ terms correspond to the 8 possible combinations of positive and negative frequency of the $k, p$ and $q$ four-momenta. Thus, we have

$$
\begin{aligned}
\mathcal{S}_{+++} & =\mathcal{P}_{T}^{00|00| 00}\left(k_{+}, p_{+}, q_{+}\right)\left[1+n_{T}\left(\varepsilon_{\mathbf{k}}\right)\right]\left[1+n_{T}\left(\varepsilon_{\mathbf{p}}\right)\right]\left[1+n_{T}\left(\varepsilon_{\mathbf{q}}\right)\right] \\
\mathcal{S}_{-++} & =\mathcal{P}_{T}^{00|00| 00}\left(k_{-}, p_{+}, q_{+}\right) n_{T}\left(\varepsilon_{\mathbf{k}}\right)\left[1+n_{T}\left(\varepsilon_{\mathbf{p}}\right)\right]\left[1+n_{T}\left(\varepsilon_{\mathbf{q}}\right)\right] \\
& \ldots \\
\mathcal{S}_{---} & =\mathcal{P}_{T}^{00|00| 00}\left(k_{-}, p_{-}, q_{-}\right) n_{T}\left(\varepsilon_{\mathbf{k}}\right) n_{T}\left(\varepsilon_{\mathbf{p}}\right) n_{T}\left(\varepsilon_{\mathbf{q}}\right) .
\end{aligned}
$$

where $k_{ \pm}= \pm \varepsilon_{\mathbf{k}}$, and similarly for $p$ and $q$. We can then integrate in $\mathbf{p}$ and $\mathbf{q}$ to get:

$$
k_{t}(T)=-\frac{1}{(2 \pi)^{3}} \int \mathrm{d}^{3} \mathrm{k} \frac{1}{8 \varepsilon_{\mathbf{k}}} \frac{\partial^{2}}{\partial p_{3} \partial q_{3}}\left[\frac{1}{\varepsilon_{\mathbf{p}} \varepsilon_{\mathbf{q}}}\left(\mathcal{S}_{+++}+\cdots+\mathcal{S}_{---}\right)\right]_{\mathbf{p}=-\mathbf{k}, \mathbf{q}=\mathbf{k}} .
$$


TABLE I: The integrand functions $I(k, m, T)$ (see eq. 400$)$ for the correlators in 300 of a free real scalar field.

\begin{tabular}{ll}
\hline \hline & $I(k, m, T)$ \\
\hline$k_{t}$ & $\frac{k^{2}}{96 T^{2} \varepsilon_{k}} \sinh ^{-6}\left(\frac{\varepsilon_{k}}{2 T}\right) \sinh \left(\frac{\varepsilon_{k}}{T}\right)\left\{k^{2} \varepsilon_{k}^{2}+T^{2}\left[k^{2}+3 \varepsilon_{k}^{2}(1-4 \xi)\right]\left[\cosh \left(\frac{\varepsilon_{k}}{T}\right)-1\right]-2 T k^{2} \varepsilon_{k} \sinh \left(\frac{\varepsilon_{k}}{T}\right)\right\}$ \\
$k_{\theta}$ & $\frac{k^{2}}{288 T^{2} \varepsilon_{k}} \sinh ^{-6}\left(\frac{\varepsilon_{k}}{2 T}\right) \sinh \left(\frac{\varepsilon_{k}}{T}\right)\left\{k^{4}+3 T^{2}\left[k^{2}(1-4 \xi)+\varepsilon_{k}^{2}(8 \xi-1)\right]\left[\cosh \left(\frac{\varepsilon_{k}}{T}\right)-1\right]-2 T k^{2} \varepsilon_{k} \sinh \left(\frac{\varepsilon_{k}}{T}\right)\right\}$ \\
$k_{s}$ & $\frac{k^{2}}{480 T^{2} \varepsilon_{k}} \sinh ^{-6}\left(\frac{\varepsilon_{k}}{2 T}\right) \sinh \left(\frac{\varepsilon_{k}}{T}\right)\left\{k^{2} \varepsilon_{k}^{2}+15 T^{2} \varepsilon_{k}^{2}(1-2 \xi)\left[\cosh \left(\frac{\varepsilon_{k}}{T}\right)-1\right]-5 T k^{2} \varepsilon_{k} \sinh \left(\frac{\varepsilon_{k}}{T}\right)\right\}$ \\
$j_{t}$ & $\frac{k^{4}}{24 \varepsilon_{k}} \sinh ^{-4}\left(\frac{\varepsilon_{k}}{2 T}\right) \sinh \left(\frac{\varepsilon_{k}}{T}\right)(1-4 \xi)$ \\
$j_{\theta}$ & $\frac{k^{4}}{72 \varepsilon_{k}} \sinh ^{-4}\left(\frac{\varepsilon_{k}}{2 T}\right) \sinh \left(\frac{\varepsilon_{k}}{T}\right)(8 \xi-1)$ \\
$j_{s}$ & $\frac{k^{4}}{48 \varepsilon_{k}} \sinh ^{-4}\left(\frac{\varepsilon_{k}}{2 T}\right) \sinh \left(\frac{\varepsilon_{k}}{T}\right)(2 \xi-1)$ \\
$l_{v}$ & $\frac{k^{4}}{24 T \varepsilon_{k}} \sinh ^{-3}\left(\frac{\varepsilon_{k}}{2 T}\right) \cosh \left(\frac{\varepsilon_{k}}{2 T}\right)\left[2 T(2 \xi-1)+\varepsilon_{k} \operatorname{coth}\left(\frac{\varepsilon_{k}}{2 T}\right)\right]$ \\
\hline \hline
\end{tabular}

TABLE II: The correlators (30) calculated for a free real scalar field with vanishing chemical potential. Also shown the well-known expressions of $\rho$ and $p$.

\begin{tabular}{lrl}
\hline \hline & $\kappa(\xi)$ & $a_{r}(x, \xi)$ \\
\hline$\rho$ & $\frac{1}{30} \pi^{2}$ & $-(r x)^{-2} K_{2}(r x)+(r x)^{-1} K_{3}(r x)$ \\
$p$ & $\frac{1}{90} \pi^{2}$ & $(r x)^{-2} K_{2}(r x)$ \\
$k_{t}$ & $-\frac{1}{30} \pi^{2}+\frac{1}{6}-\xi$ & $\frac{1}{12}\left\{\left[r^{2}-1+24 \xi x^{-2}\right] K_{2}(r x)+3\left[r(1-8 \xi)-3 r^{-1}\right] x^{-1} K_{3}(r x)\right\}$ \\
$k_{\theta}$ & $-\frac{1}{90} \pi^{2}-\frac{1}{18}+\frac{1}{3} \xi$ & $\frac{1}{12}\left\{8(1-5 \xi) x^{-2} K_{2}(r x)+\left[r(16 \xi-3)-3 r^{-1}\right] x^{-1} K_{3}(r x)\right\}$ \\
$k_{s}$ & $-\frac{1}{90} \pi^{2}+\frac{1}{12}-\frac{1}{2} \xi$ & $-\frac{1}{4}\left\{2(1-2 \xi) x^{-2} K_{2}(r x)+\left[r(4 \xi-1)+r^{-1}\right] x^{-1} K_{3}(r x)\right\}$ \\
$j_{t}$ & $\frac{1}{6}(1-4 \xi)$ & $(1-4 \xi) x^{-2} K_{2}(r x)$ \\
$j_{\theta}$ & $\frac{1}{18}(8 \xi-1)$ & $\frac{1}{3}(8 \xi-1) x^{-2} K_{2}(r x)$ \\
$j_{s}$ & $\frac{1}{12}(2 \xi-1)$ & $\frac{1}{2}(2 \xi-1) x^{-2} K_{2}(r x)$ \\
$l_{v}$ & $\frac{1}{135} \pi^{2}+\frac{1}{18}+\frac{1}{3} \xi$ & $\frac{1}{6}\left[(12 \xi-6) x^{-2} K_{2}(r x)+\left(2 r+r^{-1}\right) x^{-1} K_{3}(r x)\right]$ \\
\hline \hline
\end{tabular}

All the correlators in eq. (30) can be calculated in a similar fashion although it should be pointed out that the case of $k_{t}$ is somewhat simpler because in eq. (38) only one term in eq. (37) survived. Indeed, in general, one can have up to four terms associated with different sets of indices. Thus, the general correlator can be expressed as an integral of a sum of terms analogous to that appearing in eq. (39).

In the massless case, $T$ is the only energy scale and, on purely dimensional grounds, one finds that the correlators are of the form $\kappa(\xi) T^{4}$. For instance, integrating the eq. (39) with $m=0$ one obtains:

$$
k_{t}(T)=\left(-\frac{1}{30} \pi^{2}+\frac{1}{6}-\xi\right) T^{4} .
$$

For the massive case, the integration is just a little more involved. First, the angular part of the integration in $\mathbf{k}$ can be readily carried out and one is left with expressions like:

$$
\frac{1}{2 \pi^{2}} \int_{0}^{\infty} \mathrm{d} k I(k, m, T) .
$$

where the function $I(k, m, T)$ is reported in table Ifor the various correlators. The integral in eq. 40 can be computed setting $k=m \sinh y$, which makes it possible to extract a $m^{4}$ factor; the integral then depends on $m$ and $T$ only through the ratio $x=m / T$ and one is then left with an adimensional integral over $y$ that can be turned into a series of type (41) involving the modified Bessel functions of the second type $K_{n}(x)$ :

$$
\frac{m^{4}}{2 \pi^{2}} \sum_{r=1}^{\infty} a_{r}(x, \xi),
$$

where, as has been mentioned, $x=m / T$. The final expression of functions $a$ and $\kappa$ can be found in table II 
TABLE III: The coefficients of the stress-energy tensor in eq. (34) calculated for a free real scalar field with vanishing chemical potential.

\begin{tabular}{crlr}
\hline \hline & $\kappa(\xi)$ & $a_{r}(x, \xi)$ & $f(m, t)$ \\
\hline$U_{\alpha}$ & $\frac{1}{12}(1-6 \xi)$ & $\frac{1}{24}\left[\left(r^{2}+24 \xi x^{-2}\right) K_{2}(r x)+3(1-8 \xi) r x^{-1} K_{3}(r x)\right]$ & $\frac{1}{24} m^{2} T^{-1}+\frac{1}{8} m(1-8 \xi)+\left(\frac{5}{16}-\frac{3}{2} \xi\right) T+o(T)$ \\
$U_{w}$ & $\frac{1}{12}(1-4 \xi)$ & $\frac{1}{2}(1-4 \xi) x^{-2} K_{2}(r x)$ & $\left(\frac{1}{2}-2 \xi\right) T+o(T)$ \\
$D_{\alpha}$ & $\frac{1}{18}(6 \xi-1)$ & $\frac{1}{24}\left[(12-48 \xi) x^{-2} K_{2}(r x)+(24 \xi-5) r x^{-1} K_{3}(r x)\right]$ & $m\left(\xi-\frac{5}{24}\right)+\left(\frac{1}{2} \xi-\frac{1}{48}\right) T+o(T)$ \\
$D_{w}$ & $\frac{1}{6} \xi$ & $\xi x^{-2} K_{2}(r x)$ & $\xi T+o(T)$ \\
$A$ & $\frac{1}{12}(1-6 \xi)$ & $\frac{1}{4}\left[(4 \xi-2) x^{-2} K_{2}(r x)+(1-4 \xi) r x^{-1} K_{3}(r x)\right]$ & $m\left(\frac{1}{4}-\xi\right)+\left(\frac{1}{8}-\frac{3}{2} \xi\right) T+o(T)$ \\
$W$ & $\frac{1}{12}(2 \xi-1)$ & $\frac{1}{2}(2 \xi-1) x^{-2} K_{2}(r x)$ & $\left(\xi-\frac{1}{2}\right) T+o(T)$ \\
$G$ & $\frac{1}{36}(1+6 \xi)$ & $\frac{1}{6}\left[(6 \xi-3) x^{-2} K_{2}(r x)+r x^{-1} K_{3}(r x)\right]$ & $\frac{1}{6} m+\left(\xi-\frac{1}{12}\right) T+o(T)$ \\
\hline \hline
\end{tabular}

With the correlators calculated, we are now in a position to write down the coefficients of eq. (32), reported in table III alongside with their non-relativistic limit $m / T=x \gg 1$, factorized as $n f(m, T)$ where:

$$
n=\frac{m^{3}}{2 \pi^{2}} \sum_{r=1}^{\infty}(r x)^{-1} K_{2}(r x)
$$

is the particle density at the homogeneous equilibrium. The non-relativistic limit can be extracted by simply taking the asymptotic expansion of the $r=1$ term of each series.

As it can be seen from the table III, all the coefficients $U, A, D, W, G$ have a finite non-relativistic limit with the dimension of an energy per unit volume. Consequently, as it has been mentioned, all the corrections to the stressenergy tensor in eq. (34) are of quantum origin as they linearly depend on $\hbar$.

The coefficient $W=\lambda_{3} / T^{2}$ for the massless case turns out to be in agreement with the calculation in ref. [6] for $\xi=0$. However, unlike therein argued, we found that it has an explicit dependence on $\xi$, that is on the stress-energy tensor form.

\section{THERMODYNAMICAL INEQUIVALENCE, FRAME DEPENDENCE AND EQUATION OF STATE}

We are now going to discuss some physical consequences of the general form of the stress-energy tensor (34) which we rewrite here:

$$
\begin{aligned}
T^{\mu \nu}(x)= & {\left[\rho+\bar{a}^{2} U_{\alpha}+\bar{\omega}^{2} U_{w}\right] u^{\mu} u^{\nu}-\left[p+\bar{a}^{2} D_{\alpha}+\bar{\omega}^{2} D_{w}\right] \Delta^{\mu \nu} } \\
& +A \bar{a}^{2} \hat{a}^{\mu} \hat{a}^{\nu}+W \bar{\omega}^{2} \hat{\omega}^{\mu} \hat{\omega}^{\nu}+G \bar{a} \bar{\omega}\left(u^{\mu} \hat{\gamma}^{\nu}+\hat{\gamma}^{\mu} u^{\nu}\right)+o\left(\varpi^{2}\right)
\end{aligned}
$$

where the shorthands $\bar{a}=\hbar|a| / c K T$ and $\bar{\omega}=\hbar|a| / K T$ for the adimensional scales related to acceleration and vorticity.

The first remarkable consequence is that, as pointed out in refs. 21, 22, the mean stress-energy tensor in a general thermodynamic equilibrium depends on the fundamental stress-energy tensor operator written in terms of the quantum fields. This is at variance with the familiar homogeneous equilibrium, and it is made apparent by the dependence of the thermal functions other than $\rho$ and $p$ in table III on the parameter $\xi$. If one was able to measure one of the coefficients multiplying $\bar{a}^{2}$ or $\bar{\omega}^{2}$ with a thermodynamics experiment, one would obtain information about the true, physical stress-energy tensor operator, hence on the correct gravitational theory, a conclusion already drawn in ref. [21].

The second consequence is that, as it is apparent from the eq. $443, u^{\nu}=T \beta^{\nu}$ is not an eigenvector of $T^{\mu \nu}$ if $\gamma$ is non-vanishing, that is if the three vectors $\alpha, w, u$ (or $a, \omega, u)$ are linearly independent, as it can be seen from the eq. (34). This is what happens for the the rigid rotation, where $a, \omega$ and $u$ are orthogonal to each other. In this case, the $u$ vector does not coincide with the Landau definition of four-velocity, and should then be taken as defining a new hydrodynamical frame, dubbed the $\beta$ frame, as it has been extensively discussed in ref. [9].

The third, and perhaps the most striking consequence, is that the dependence of energy density and pressure on the temperature and chemical potential are modified with respect to the homogeneous equilibrium case. Also, there are more second-order coefficients in the expansion of the stress-energy tensor than previously envisaged. Looking at the eq. (43) it can be realized that, with respect to the expansions presented in refs. [3, 4, 6, there are three new 
coefficients, that is $G, U_{\alpha}, U_{w}$ and two of them imply a modification of the energy density. One could argue that they would disappear by going to the Landau frame. Yet, in the diagonalization of the stress-energy tensor in eq. (43), it can be readily shown that, retaining only quadratic terms in $\bar{a}$ and $\bar{\omega}$ :

$$
\begin{aligned}
& \rho_{\text {eff }}=\rho+\bar{a}^{2} U_{\alpha}+\bar{\omega}^{2} U_{w}+o\left(\varpi^{2}\right) \\
& p_{\text {eff }}=p+\bar{a}^{2}\left(D_{\alpha}+\frac{1}{3} A\right)+\bar{\omega}^{2}\left(D_{w}+\frac{1}{3} W\right)+o\left(\varpi^{2}\right),
\end{aligned}
$$

where the effective pressure has been defined as the mean of the eigenvalues of the spacelike eigenvectors. Therefore, the energy density and the pressure coincide, in this approximation, with those in the $\beta$ frame and the coefficients $U_{\alpha}$ and $U_{w}$ survive. One may wonder whether the modification of the energy density could be reabsorbed by a redefinition of the temperature other than the length of the $\beta$ vector in the density operator in the eq. (6), which is based on the maximization of entropy with macroscopic constraints [9]. In fact, a redefinition would cure only one of the eigenvalues of the stress-energy tensor, unless the coefficients $U, D, A, W$ fulfilled some preculiar relations. In all other cases, the relation between the eigenvalues of the stress-energy tensor, or the relation between energy density and pressure, in other words the equation of state $p_{\text {eff }}\left(\rho_{\text {eff }}\right)$, is modified with respect to the homogeneous equilibrium case. For instance, in the non-relativistic limit of the massive case $m \gg T$ one has, according to table III that the leading corrections are those in $\bar{a}^{2}$, and restoring the natural constants:

$$
\begin{aligned}
& \rho_{\mathrm{eff}} \simeq \rho+\frac{1}{24} \frac{m c^{2}}{K T} \rho \bar{a}^{2}=\left(1+\frac{1}{24} \frac{m \hbar^{2}|a|^{2}}{(K T)^{3}}\right) \rho \\
& p_{\mathrm{eff}} \simeq p+\left(\frac{2}{3} \xi-\frac{1}{8}\right) m c^{2} \bar{a}^{2} n=p\left[1+\left(\frac{2}{3} \xi-\frac{1}{8}\right) \frac{m \hbar^{2}|a|^{2}}{(K T)^{3}}\right]
\end{aligned}
$$

where $\rho=m n$ and $p=n K T$ are the usual non-relativistic expressions for the ideal Boltzmann gas and $n$ has the well known approximate expression:

$$
n \simeq\left(\frac{m T}{2 \pi}\right)^{3 / 2} \mathrm{e}^{-m / T}
$$

We note in passing that the relations (45) should hold in the case of a charged scalar field in the non-degenerate Boltzmann limit with a chemical potential, that is:

$$
n \simeq\left(\frac{m T}{2 \pi}\right)^{3 / 2} \mathrm{e}^{(\mu-m) / T}
$$

and negligible anti-particle contribution.

If it was possible to redefine $T$ to a new $T^{\prime}=T+b(T) \bar{a}^{2}$ such that $\rho=m n\left(T^{\prime}\right)$ and $p=T^{\prime} n\left(T^{\prime}\right)$, then the coefficients in the $\bar{a}^{2}$ expansion of the functions would be the same. This can be shown by taking into account that $\partial n / \partial T \simeq\left(m / T^{2}\right) n(T)$ in the non-relativistic $m \gg T$ limit, so that

$$
\begin{aligned}
& \rho\left(T^{\prime}\right)=m n\left(T^{\prime}\right) \simeq m n(T)+\frac{\partial n}{\partial T}\left(T^{\prime}-T\right)=m n(T)\left(1+\frac{m}{T^{2}} b \bar{a}^{2}\right) \\
& p\left(T^{\prime}\right)=T^{\prime} n\left(T^{\prime}\right) \simeq T n(T)+n(T)\left(1+\frac{m}{T}\right) b \bar{a}^{2} \simeq T n(T)\left(1+\frac{m}{T^{2}} b \bar{a}^{2}\right)
\end{aligned}
$$

However, it can be seen by comparing the above equation with 45 that in general this is not the case, except when $\xi=1 / 4$ which is neither the canonical nor the improved tensor.

Furthermore, in general, the redefinition of a temperature would be mass dependent and it would then be troublesome to define thermodynamic equilibrium at a common temperature of a mixture of gases. Let

$$
\rho_{\mathrm{eff}}(T, \bar{a}, \bar{\omega})=\rho\left(T^{\prime}(T, \bar{a}, \bar{\omega})\right),
$$

where $\rho$ is the familiar homogeneous energy density. Expanding the new temperature in $\bar{a}$ and $\bar{\omega}$ the leading order corrections must be of the second order:

$$
T^{\prime}=T+T_{\bar{a}}(T) \bar{a}^{2}+T_{\bar{\omega}}(T) \bar{\omega}^{2}+o\left(\varpi^{2}\right),
$$

where $T_{\bar{a}}$ and $T_{\bar{\omega}}$ are proportional to the second derivatives of $T^{\prime}(T, \bar{a}, \bar{\omega})$ with respect to $\bar{a}$ and $\bar{\omega}$ respectively. These unknown functions can be obtained by comparing with the equation 44 :

$$
\rho+\frac{\partial \rho}{\partial T}\left(T_{\bar{a}} \bar{a}^{2}+T_{\bar{\omega}} \bar{\omega}^{2}\right)=\rho+\bar{a}^{2} U_{\alpha}+\bar{\omega}^{2} U_{w}+o\left(\varpi^{2}\right),
$$


implying

$$
T^{\prime}=T+\frac{U_{\alpha}}{\partial \rho / \partial T} \bar{a}^{2}+\frac{U_{w}}{\partial \rho / \partial T} \bar{\omega}^{2}+o\left(\varpi^{2}\right) .
$$

Looking at the tables (II) and (III), it can be realized that the coefficients of $\bar{a}^{2}$ and $\bar{\omega}^{2}$ are non-trivial functions of the mass and temperature.

Going now back to the properly defined $T=1 / \sqrt{\beta^{2}}$, we observe that, in the non-relativistic limit the relation between the effective energy density and pressure gets modified into:

$$
p_{e f f} \simeq \rho_{e f f} \frac{K T}{m}\left[1+\left(\frac{2}{3} \xi-\frac{1}{6}\right) \frac{m \hbar^{2}|a|^{2}}{(K T)^{3}}\right]
$$

Therefore, the effective equation of state depends on the acceleration besides the temperature. This could be surprising, but in fact in general global equilibrium all parameters, including acceleration and angular velocity play the role of thermodynamic variables on the same footing as temperature and chemical potential. It can be seen that in the nonrelativistic non-degenerate limit the quantum correction to the relations (44) and the equation of state becomes more important at low proper temperature, being proportional to $1 / T^{3}$. Of course this applies as long as the acceleration is such that $m \hbar^{2}|a|^{2} /(K T)^{3} \ll 1$ so that the expansion method holds 3 , for very low temperatures, one would have to take more and more terms into account and eventually the exact solution would be needed.

\section{CONCLUSIONS}

In conclusion, we have demonstrated that the relativistic stress-energy tensor in general states of global thermodynamic equilibrium features quantum corrections with respect to its ideal form (2) depending on the local values of acceleration and vorticity, besides proper temperature and chemical potential. We have calculated the coefficients of the additional terms of the stress-energy tensor in the appropriate quantum statistical framework at the second order of an expansion in the parameters $\hbar a / c K T$ and $\hbar \omega / K T$ for the simplest case of a real scalar field. We have found that more terms exist with respect to previous assessments; our calculated coefficient $W$ for the real scalar field agrees with previous calculations [6].

We have emphasized three major physical consequences of this finding:

1. The coefficients explicitely depend on the form of the quantum stress-energy tensor operator, what was already argued in refs. [21, 22].

2. The effective energy density - defined as the eigenvalue of the stress-energy tensor - is also modified by terms involving acceleration and vorticity which cannot be reabsorbed by means of a redefinition of the temperature.

3. The equation of state and the relation between effective pressure and effective energy density are also modified by the presence of vorticity and acceleration.

In principle, these findings could be extended to matter in local thermodynamic equilibrium in flat spacetime, as well as to matter in global/local equilibrium in a curved spacetime. In this case, it is well known that $\beta$ in eq. (6) must be a Killing vector which can have a non-vanishing exterior derivative $\partial_{\mu} \beta_{\nu}-\partial_{\nu} \beta_{\mu}$ and, consequently, additional terms of the stress-energy tensor with respect to its ideal form (2). This might be of phenomenological relevance for the study of the equilibrium of self-gravitating objects.

\section{ACKNOWLEDGMENTS}

We are greatly indebted to R. Panerai for numerous suggestions and help in calculations. We acknowledge interesting discussions with S. Capozziello, N. Pinamonti and P. Romatschke.

\section{REFERENCES}

[1] L. Landau, L. Lifshitz, Statistical Physics, Pergamon Press (1980).

\footnotetext{
${ }^{3}$ For a proton and $|a|=g$ one has that the ratio becomes $\mathcal{O}(1)$ for $T \approx 10^{-8}$ kelvin
} 
[2] A. Vilenkin, Phys. Rev. D 212260 (1980).

[3] R. Baier, P. Romatschke, D. T. Son, A. O. Starinets and M. A. Stephanov, JHEP 0804, 100 (2008).

[4] P. Romatschke, Class. Quant. Grav. 27, 025006 (2010)

[5] G. S. Denicol, H. Niemi, E. Molnar and D. H. Rischke, Phys. Rev. D 85, 114047 (2012) [Erratum-ibid. D 91, no. 3, 039902 (2015)]

[6] G. D. Moore and K. A. Sohrabi, JHEP 1211, 148 (2012).

[7] D. N. Zubarev, A. V. Prozorkevich, S. A. Smolyanskii, Theoret. and Math. Phys. 40 (1979), 821.

[8] Ch. G. Van Weert, Ann. Phys. 140, 133 (1982).

[9] F. Becattini, L. Bucciantini, E. Grossi and L. Tinti, arXiv:1403.6265 [hep-th].

[10] T. Hayata, Y. Hidaka, M. Hongo and T. Noumi, arXiv:1503.04535[hep-ph].

[11] F. Becattini, Phys. Rev. Lett. 108, 244502 (2012).

[12] R. D. de Souza, T. Koide and T. Kodama, arXiv:1506.03863 [nucl-th].

[13] P. Arnold, D. Vaman, C. Wu and W. Xiao, JHEP 1110, 033 (2011).

[14] S. Grozdanov and A. O. Starinets, JHEP 1503, 007 (2015)

[15] S. I. Finazzo, R. Rougemont, H. Marrochio and J. Noronha, JHEP 1502, 051 (2015).

[16] O. Philipsen and C. Schäfer, JHEP 1402, 003 (2014).

[17] E. Molnar, H. Niemi, G. S. Denicol and D. H. Rischke, Phys. Rev. D 89, no. 7, 074010 (2014).

[18] W. Florkowski, A. Jaiswal, E. Maksymiuk, R. Ryblewski and M. Strickland, Phys. Rev. C 91054907 (2015).

[19] C. G. Callan, Jr., S. R. Coleman and R. Jackiw, Ann. Phys. 59, 42 (1970).

[20] T. S. Evans and D. A. Steer, Nucl. Phys. B 474, 481 (1996).

[21] F. Becattini and L. Tinti, Phys. Rev. D 84, 025013 (2011).

[22] F. Becattini and L. Tinti, Phys. Rev. D 87, 025029 (2013).

\section{Appendix A: $\widehat{\mathcal{R}}$ expansion}

To derive the espressions of $\widehat{\mathcal{R}}^{(n)}$, we can disregard, for the sake of simplicity, the conserved charge in 22 for it commutes with both $\widehat{P}$ and $\widehat{J}$ operators. Defining

$$
\widehat{A}=-\beta_{\mu} \widehat{P}^{\mu} \quad \widehat{B}=\frac{1}{2} \varpi_{\mu \nu} \widehat{J}^{\mu \nu},
$$

and applying the known Poincaré algebra relations, we find:

$$
\begin{aligned}
{[\widehat{A}, \widehat{B}] } & =-i \beta^{\mu} \varpi_{\mu \nu} \widehat{P}^{\nu}, \\
{[[\widehat{A}, \widehat{B}], \widehat{A}] } & =0 \\
{[[\widehat{A}, \widehat{B}], \widehat{B}] } & =\beta^{\mu} \varpi_{\mu \nu} \omega_{\rho \sigma} g^{\nu \rho} \widehat{P}^{\sigma}, \\
{[[[[\widehat{A}, \widehat{B}], \widehat{B}], \ldots], \widehat{B}] } & =-(i)^{n} \beta^{\mu}(\varpi \cdot \varpi \cdot \ldots \cdot \varpi)_{\mu \nu} \widehat{P}^{\nu}
\end{aligned}
$$

Now, using the Baker-Campbell-Hausdorff formula to expand $\exp [\widehat{A}+\widehat{B}]$ and retaining only the non-vanishing terms; taking into account that any commutator involving $\widehat{A}, \widehat{B}$ or commutators thereof, will in turn commute with $\widehat{A}$, being proportional to four-momentum operators, we obtain:

$$
\begin{aligned}
\widehat{\mathcal{R}}(\beta, \varpi)=\mathrm{e}^{\widehat{A}+\widehat{B}} & \simeq \mathrm{e}^{\widehat{B}} \mathrm{e}^{\widehat{A}} \mathrm{e}^{\frac{1}{2 !}[\widehat{A}, \widehat{B}]} \mathrm{e}^{\frac{1}{3 !}[[\widehat{A}, \widehat{B}], \widehat{B}]} \mathrm{e}^{\frac{1}{4 !}[[[\widehat{A}, \widehat{B}], \widehat{B}], \widehat{B}]} \ldots \\
& =\mathrm{e}^{\widehat{B}} \mathrm{e}^{\widehat{A}+\frac{1}{2 !}[\widehat{A}, \widehat{B}]+\frac{1}{3 !}[[\widehat{A}, \widehat{B}], \widehat{B}]+\frac{1}{4 !}[[[\widehat{A}, \widehat{B}], \widehat{B}], \widehat{B}]+\ldots}
\end{aligned}
$$

and its expansion up to second order in $\widehat{B}$ (which is tantamount to a second order in $\varpi$ ) reads:

$$
\widehat{\mathcal{R}}(\beta, \varpi) \simeq \mathrm{e}^{\widehat{A}}+\left(\widehat{B}+\frac{1}{2}[\widehat{A}, \widehat{B}]\right) \mathrm{e}^{\widehat{A}}+\left(\frac{1}{2} \widehat{B}^{2}+\frac{1}{3} \widehat{B}[\widehat{A}, \widehat{B}]+\frac{1}{6}[\widehat{A}, \widehat{B}] \widehat{B}+\frac{1}{8}[\widehat{A}, \widehat{B}]^{2}\right) \mathrm{e}^{\widehat{A}}
$$

where advantage has been taken of the fact that $\exp [\widehat{A}]$ commutes with both the commutators $[\widehat{A}, \widehat{B}]$ and $[[\widehat{A}, \widehat{B}], \widehat{B}]$. Now, by using the relation:

$$
\mathrm{e}^{-\widehat{A}} \widehat{B} \mathrm{e}^{\widehat{A}}=\widehat{B}-[\widehat{A}, \widehat{B}]
$$

which is a known corollary of the Baker-Campbell-Hausdorff formula for our case, the eq. A3 can be rewritten as:

$$
\widehat{\mathcal{R}}(\beta, \varpi) \simeq \mathrm{e}^{\widehat{A}}+\mathrm{e}^{\widehat{A}}\left(\widehat{B}-\frac{1}{2}[\widehat{A}, \widehat{B}]\right)+\mathrm{e}^{\widehat{A}}\left(\frac{1}{2} \widehat{B}^{2}-\frac{1}{6} \widehat{B}[\widehat{A}, \widehat{B}]-\frac{1}{3}[\widehat{A}, \widehat{B}] \widehat{B}+\frac{1}{8}[\widehat{A}, \widehat{B}]^{2}\right)
$$


We can now take the half-sum of A3 and A4 to obtain:

$$
\widehat{\mathcal{R}}(\beta, \varpi) \simeq \mathrm{e}^{\widehat{A}}+\frac{1}{2}\left\{\mathrm{e}^{\widehat{A}}, \widehat{B}\right\}+\frac{1}{4}\left\{\mathrm{e}^{\widehat{A}}, \widehat{B}^{2}\right\}-\frac{1}{8} \mathrm{e}^{\widehat{A}}[\widehat{A}, \widehat{B}]^{2}-\frac{1}{12} \mathrm{e}^{\widehat{A}}[[\widehat{A}, \widehat{B}], \widehat{B}]
$$

putting the expressions of $\widehat{A}$ and $\widehat{B}$ in eq. A1 in the eq. $\mathrm{A} 5$ one can read off the operators in eq. 23., which are quoted in eq. (24).

\section{Appendix B: Calculation of angular momentum-stress energy tensor correlators}

The density operator 11 , which is used to calculate the mean values denoted as \langle\rangle$_{\beta}$ can be written as $\widehat{\Lambda} \rho_{0} \widehat{\Lambda}-1$ where $\widehat{\Lambda}$ is the Lorentz transformation turning $\beta_{0}=(1 / T, \mathbf{0})$ into $\beta$. Hence, the mean value of a general tensor can be expanded as:

$$
\left\langle\widehat{O}^{\mu_{1}, \ldots, \mu_{N}}\right\rangle_{\beta(x)}=\Lambda_{\nu_{1}}^{\mu_{1}} \ldots \Lambda_{\nu_{N}}^{\mu_{N}}\left\langle\widehat{O}^{\nu_{1}, \ldots, \nu_{N}}\right\rangle_{T}
$$

where \langle\rangle$_{T}$, as has been mentioned in the text, stands for the mean value with the density operator $\frac{1}{Z} \exp \left[-\beta_{0} \cdot \widehat{P}\right]=$ $\frac{1}{Z} \exp [-\widehat{H} / T]$. Note that:

$$
\Lambda_{0}^{\mu}=\hat{\beta}^{\mu} \quad \sum_{i, j=1}^{3} \Lambda_{i}^{\mu} \Lambda_{j}^{\nu} g^{i j}=g^{\mu \nu}-\hat{\beta}^{\mu} \hat{\beta}^{\nu}=\Delta^{\mu \nu}
$$

Since $\exp [-\widehat{H} / T]$ is invariant by rotation, only scalars under spatial rotation, either components or contractions of the tensor $O^{\nu_{1}, \ldots, \nu_{N}}$ may have a non vanishing value. Furthermore, we assume that the hamiltonian operator is symmetric under parity and time reversal transformations, so that also pseudoscalars and scalars which are odd under time reversal will have vanishing mean value.

For instance, for a symmetric tensor operator $\widehat{S}^{\mu \nu}$ one can write:

$$
\left\langle\widehat{S}^{\mu \nu}\right\rangle_{T}=\delta_{0}^{\mu} \delta_{0}^{\nu} A+\tilde{\Delta}^{\mu \nu} B
$$

where $\tilde{\Delta}^{\mu \nu}$ is the transverse projector in the rest frame, i.e. $\tilde{\Delta}^{\mu \nu}=g^{\mu \nu}-\delta_{0}^{\mu} \delta_{0}^{\nu}$. Of course, the eq. B3) becomes the well known:

$$
\left\langle\widehat{S}^{\mu \nu}\right\rangle_{\beta}=A \hat{\beta}^{\mu} \hat{\beta}^{\nu}+\Delta^{\mu \nu} B
$$

by using the (B1) and $(\mathrm{B} 2)$. The coefficients $A$ and $B$ can be calculated from the mean values selecting the components in eq. (B3) which make all terms vanishing except one. Thereby:

$$
A=\left\langle\widehat{S}^{00}\right\rangle_{T} \quad B=-\left\langle\widehat{S}^{i i}\right\rangle_{T}
$$

This general procedure can be applied to the calculations of tensors of any rank. Indeed, in view of eq. (B2), anytime a time component or 0 index is selected in $\left\langle\widehat{O}^{\nu_{1}, \ldots, \nu_{N}}\right\rangle_{T}$ in eq. B1 a $\delta_{0}^{\nu}$ will appear eventually turning into a $u$ after boosting, while for a space contraction of indices a $\tilde{\Delta}$ projector will, eventually turning into a $\Delta$ like in eq. (B2).

We can first apply the above argument to the calculation of $\left\langle\widehat{J}^{\mu \nu} \widehat{T}^{\rho \sigma}\right\rangle_{\beta}$. By using the decomposition $(27)$ and taking into account (B1), the only possible non-vanishing contributions read:

$$
\operatorname{Re}\left\langle\widehat{K}_{i} \widehat{T}^{0 i}\right\rangle_{T} \quad \operatorname{Re}\left\langle\widehat{J}_{i} \widehat{T}^{0 i}\right\rangle_{T}
$$

Yet, they both vanish because they are odd under time reversal and parity respectively. No scalar can be formed with $\left\langle\widehat{J}^{\mu \nu}\right\rangle_{T}$ and so the mean value of the angular momentum $\left\langle\widehat{J}^{\mu \nu}\right\rangle_{\beta}$ vanishes too.

Let us now move to the more complicated case of correlators involving two angular momentum operators, starting from:

$$
\left\langle\left\{\widehat{K}^{\rho}, \widehat{K}^{\sigma}\right\} ; \widehat{T}^{\mu \nu}\right\rangle_{T}
$$


In the rest frame, $\left\{\widehat{K}^{\rho}, \widehat{K}^{\sigma}\right\}$ is a symmetric tensor with vanishing time components, so it has one spin-0 component obtained with the contraction of the indices $\rho$ and $\sigma$ and one spin-2 component under rotation which can be obtained by applying the projector:

$$
P_{\alpha \beta}^{\rho \sigma}=\frac{1}{2}\left(\tilde{\Delta}_{\alpha}^{\rho} \tilde{\Delta}_{\beta}^{\sigma}+\tilde{\Delta}_{\alpha}^{\sigma} \tilde{\Delta}_{\beta}^{\rho}-\frac{2}{3} \tilde{\Delta}^{\rho \sigma} \tilde{\Delta}_{\alpha \beta}\right)
$$

to the tensor itself. In order to construct a rotation singlet, we need to combine the above components with the corresponding components of $\widehat{T}^{\mu \nu}$. The spin 0 components can only contract with $\widehat{T}^{00}$ and its spatial trace, so one obtains two contributions:

$$
-\tilde{\Delta}^{\rho \sigma} \delta_{0}^{\mu} \delta_{0}^{\nu} k_{t} \quad \tilde{\Delta}^{\rho \sigma} \tilde{\Delta}^{\mu \nu} k_{\theta}
$$

whereas the contraction of the spin 2 component of $\left\{\widehat{K}^{\rho}, \widehat{K}^{\sigma}\right\}$ with the one of $\widehat{T}$ gives rise to:

$$
\left(\tilde{\Delta}^{\rho \mu} \tilde{\Delta}^{\sigma \nu}+\tilde{\Delta}^{\rho \nu} \tilde{\Delta}^{\sigma \mu}-\frac{2}{3} \tilde{\Delta}^{\mu \nu} \tilde{\Delta}^{\rho \sigma}\right) k_{s}
$$

Altogether

$$
\frac{1}{2} \operatorname{Re}\left\langle\left\{\widehat{K}^{\rho}, \widehat{K}^{\sigma}\right\} ; \widehat{T}^{\mu \nu}\right\rangle_{T}=-\tilde{\Delta}^{\rho \sigma} \delta_{0}^{\mu} \delta_{0}^{\nu} k_{t}+\tilde{\Delta}^{\rho \sigma} \tilde{\Delta}^{\mu \nu} k_{\theta}+\left(\tilde{\Delta}^{\rho \mu} \tilde{\Delta}^{\sigma \nu}+\tilde{\Delta}^{\rho \nu} \tilde{\Delta}^{\sigma \mu}-\frac{2}{3} \tilde{\Delta}^{\mu \nu} \tilde{\Delta}^{\rho \sigma}\right) k_{s}
$$

which in the observer frame reads:

$$
\frac{1}{2} \operatorname{Re}\left\langle\left\{\widehat{K}^{\rho}, \widehat{K}^{\sigma}\right\} ; \widehat{T}^{\mu \nu}\right\rangle_{\beta}=-\Delta^{\rho \sigma} u^{\mu} u^{\nu} k_{t}+\Delta^{\rho \sigma} \Delta^{\mu \nu} k_{\theta}+\left(\Delta^{\rho \mu} \Delta^{\sigma \nu}+\Delta^{\rho \nu} \Delta^{\sigma \mu}-\frac{2}{3} \Delta^{\mu \nu} \Delta^{\rho \sigma}\right) k_{s}
$$

To find a compact expression of the coefficients $k_{t}, k_{\theta}, k_{s}$ one can select the indices making all terms on the right hand side of (B4) vanishing except the one of interest. One can check that all indices in the definitions (30) are properly chosen (notice how in $k_{s}$ we avoided the symmetrization in $\mu \leftrightarrow \nu$ associated with the anti-commutator since we know that the anti-symmetric part will not contribute). For the $j_{t}, j_{\theta}, j_{s}$ the procedure is precisely the same outlined above with the replacement $\widehat{K} \rightarrow \widehat{J}$.

In fact, the correlator $\left\langle\left\{\widehat{K}^{\rho}, \widehat{J}^{\sigma}\right\} ; \widehat{T}^{\mu \nu}\right\rangle_{T}$ is a somewhat special case because $\left\{\widehat{K}^{\rho}, \widehat{J}^{\sigma}\right\}$ is odd under parity and time reversal. Therefore, the only non-vanishing contraction is between the two spin-1 components of the tensors $\left\{\widehat{K}^{\rho}, \widehat{J}^{\sigma}\right\}$ and $\widehat{T}^{\mu \nu}$ respectively. The spin-1 components can be obtained by means of the projectors

$$
P_{\alpha \beta}^{\rho \sigma}=-\frac{1}{2} \epsilon^{0 \rho \sigma \tau} \epsilon_{0 \alpha \beta \tau}
$$

and

$$
P_{\alpha \beta}^{\mu \nu}=\frac{1}{2} \delta_{0}^{\mu} \tilde{\Delta}_{\alpha}^{\nu} \delta_{\beta}^{0}+\delta_{0}^{\nu} \tilde{\Delta}_{\alpha}^{\mu} \delta_{\beta}^{0}
$$

respectively. Hence:

$$
\operatorname{Re}\left\langle\left\{\widehat{K}^{\rho}, \widehat{J}^{\sigma}\right\} ; \widehat{T}^{\mu \nu}\right\rangle_{T}=\left(\epsilon^{0 \rho \sigma \tau} \delta_{0}^{\mu} \tilde{\Delta}_{\tau}^{\nu}+\epsilon^{0 \rho \sigma \tau} \delta_{0}^{\mu} \tilde{\Delta}_{\tau}^{\nu}\right) l_{v}=\left(\delta_{\kappa}^{0} \epsilon^{\kappa \rho \sigma \nu} \delta_{0}^{\mu}+\delta_{\kappa}^{0} \epsilon^{\kappa \rho \sigma \mu} \delta_{0}^{\nu}\right) l_{v}
$$

which, once boosted, reads:

$$
\operatorname{Re}\left\langle\left\{\widehat{K}^{\rho}, \widehat{J}^{\sigma}\right\} ; \widehat{T}^{\mu \nu}\right\rangle_{\beta}=\left(u_{\kappa} \epsilon^{\kappa \rho \sigma \nu} u^{\mu}+u_{\kappa} \epsilon^{\kappa \rho \sigma \mu} u^{\nu}\right) l_{v}
$$

